ITFA-96-50, hep-th/9612096

\title{
Monads and D-instantons
}

\author{
Jae-Suk Park ${ }^{\dagger}$ \\ Instituut voor Theoretische Fysica \\ Universiteit van Amsterdam \\ Valckenierstraat 65, 1018 XE Amsterdam
}

\begin{abstract}
Motivated by twisted $\mathcal{N}=4$ supersymmetric Yang-Mills theory in 4 dimensions, a natural extension of the monad (ADHM) construction relevant to D-instantons is considered. We show that a family of Yang-Mills instantons can be constructed from D-instantons. We discuss some possible roles of reciprocity in D-brane physics. We conjecture the existence of universal instantons together with a generalized Fourier-Nahm transformation as an unifying framework of D-brane physics.
\end{abstract}

November, 1996

$\dagger$ e-mail: park@phys.uva.nl 


\section{Introduction}

Shortly after the discovery of the Yang-Mills instantons [1], Atiyah, Dringfeld, Hitchin and Manin (ADHM) found of a way of constructing, in principle, all instantons for any classical group [2]. The ADHM construction relates the moduli space of anti-self-dual connections on $\mathbb{R}^{4}$ to the space of monads via the Ward correspondence [3]. Subsequently, Donaldson reformulated the ADHM construction in the language holomorphic vector bundle on $\mathbb{C P}^{2}$ 四. The ADHM construction can be generalized to the Fourier-Nahm-Mukai transformation [5] [6]. For any sub group $H$ of the group of isometries of $\mathbb{R}^{4}$, this transformation gives an isometry between the moduli space of $H$-invariant instantons on $\mathbb{R}^{4}$ and the moduli space of $H^{*}$-invariant instantons on $\mathbb{R}^{* 4}$ [5] [0] [8] [9]. This isometry and its miraculous properties, originally pointed out by Corrigan and Goddard, is called the reciprocity [10].

In the recent developments of string theory D-branes play crucial roles [11] [12]. The low energy theory on the $\mathrm{D} p$-branes world volume is the dimensional reduction of $d=10$ $\mathcal{N}=1$ super-Yang-Mills theory down to $p+1$ dimensions [13] [14]. The type IIB string has $p=-1$ D-branes or D-instantons whose low energy description has an intriguing similarity with the ADHM construction of Yang-Mills instanton [14].

Motivated by some properties of a $\mathcal{N}=4$ supersymmetric Yang-Mills theory in 4dimensions [15] [16], we will consider a simple extension of the monad (ADHM) construction. We will show that such an extension is closely related to D-instantons, which are, in turn, capable of being frozen on parallel 4-dimensional Euclidian spaces to become a family of genuine Yang-Mills instantons of different gauge groups and instanton numbers. Equivalently, we get a family of the ADHM constructions. This identification suggest that reciprocity (maybe in a suitably generalized form) may have a significant role in the Dbrane physics. Indeed, Witten's description of Dirichlet p-branes is very suggestive of such a role [14]. The various D-branes may be related to certain universal instantons invariant under suitable isometry $H$. The usual description of D-branes may be obtained by doing a sort of Fourier-Nahm-Mukai transformation.

We should mention several earlier papers relating D-branes and Yang-Mills instantons. Witten showed that the type I D 5-brane is a point-like instanton [17] using his ADHM linear sigma model [18]. Douglas extend the picture and produced the instantons using certain D-branes as the probes [19]. Douglas and Moore reproduced results of Kronheimer and Nakajima on Yang-Mills instantons in ALE space [20]. They also suggested a relation between $T$-duality and reciprocity [21]. Related issues have also studied in [22] [23].

Diaconescu's paper shows some interesting relation between the ADHMN construction of monopole D-branes [24]. 
In the above mentioned cases the Yang-Mills instantons are constructed, for example, via the world-sheet theory of a D 1-brane moving in the background of a system of $\mathrm{D}$ 5-branes and 9-branes, depending on the rank of the gauge group and on the instanton numbers. Our construction will be quite different from the above approach. We will show a direct relation between D-instantons and Yang-Mills instantons. It will lead us to suggest the possibility of reversing the usual program: D-brane physics may be described by certain universal monads in unifying way.

This paper is organized as follows, In Sect. 2, we review the ADHM construction. We first recall the result of the construction and review the reformulation of Donaldson who used monads on $\mathbb{C P}^{2}$ and geometric invariant theory. In Sect. 3, we present a simple extension of Donaldson's construction. In this extended form there is no distinction between the rank of the gauge group and the instanton number. We use the natural $S^{1}$ action on the space of extended monads and study its fixed points. The usual ADHM construction can be recovered as a special limit. In Sect. 4, we show that the extended monads construction can be obtained by dimensional reduction of a $\mathcal{N}=2$ preserving perturbation of a $\mathcal{N}=4$ super-Yang-Mills theory in 4-dimensions. We show that a further perturbation to a $\mathcal{N}=1$ theory leads to a resolution of singularities. We also formulate natural matrix models related to monads. In Sect. 5, we study the relation between the extended monads, D-instantons and Yang-Mills instantons. Finally, some implications for D-brane physics are discussed.

\section{Preliminary}

This section is devoted to a brief review of the ADHM construction and related topics. The contents of this section are classical and several good reviews exist [25] [10] [26]. The main purpose is to establish our notations and provided some background for the discussions in the later sections. Most of the material here is based on [26] and [4], to which we refer for details. See also 27 for a self-contained survey and many other issues relevant to this paper.

The ADHM construction is based on the twistor method via the Ward correspondence [3] and the monads of Horrocks [28] and Barth [29] on algebraic bundles over $\mathbb{C P}^{3}$. Donaldson reformulated the ADHM construction by identifying instantons on $\mathbb{R}^{4}$ with stable holomorphic bundles over $\mathbb{C P}^{2}$ trivial along the line at infinity [4] [30]. In both constructions, the notion of monads plays the central role. Most of our paper will be algebraic, so we will recall Nahm's approach only when it is necessary. 


\subsection{The ADHM constructions}

For our purpose it is sufficient to recall the end results (Ch. (3.3) in [26]). Consider $S U(n)$ instantons with instanton number $k$. The ADHM data is given by

(i) A $k$-dimensional complex vector space $\mathcal{H}$ and a Hermitian metric and self-adjoint linear maps $T_{i}: \mathcal{H} \rightarrow \mathcal{H}$ with $i=1,2,3,4$.

(ii) An $n$-dimensional complex Hermitian vector space $E_{\infty}$ with determinant form, and a linear map $P: E_{\infty} \rightarrow \mathcal{H} \otimes S^{+}$, where $S^{+}$is the positive two-dimensional spin space of $R^{4}$.

(iii) There is surjective linear map $R_{x}$ for each $x \in R^{4} R_{x}: \mathcal{H} \otimes S^{-} \oplus E_{\infty} \rightarrow \mathcal{H} \otimes S^{+}$ by

$$
R_{x}=\left(\sum_{i=0}^{3}\left(T_{i}-x_{i} I\right) \otimes \gamma\left(e_{i}\right)^{*}\right) \oplus P,
$$

where $\gamma\left(e_{i}\right)^{*}: S^{-} \rightarrow S^{+}$are the adjoints of the maps defining the spin structure. This is the non-degeneracy condition.

Now the ADHM data describe the moduli space of instantons by the solutions, up to gauge degree of freedom, of the ADHM equations;

$$
\begin{aligned}
& {\left[T_{1}, T_{2}\right]+\left[T_{3}, T_{4}\right]=P P_{I}^{*},} \\
& {\left[T_{1}, T_{3}\right]+\left[T_{4}, T_{2}\right]=P P_{J}^{*},} \\
& {\left[T_{1}, T_{4}\right]+\left[T_{2}, T_{3}\right]=P P_{K}^{*},}
\end{aligned}
$$

The ADHM data $\left(T_{i}, P\right)$ is equivalent to the data $\left(T_{i}^{\prime}, P^{\prime}\right)$ if

$$
T_{i}^{\prime}=v T_{i} v^{-1}, \quad P^{\prime}=v P u^{-1},
$$

for $v$ in $U(k)$ and $u$ in $S U(n)$

From the ADHM data $(T, P)$, one can reconstruct the explicit connection $A(T, P)$ by the standard way of introducing a connection via a projection onto a sub-bundle embedded in a trivial bundle with fibre $\mathcal{H} \oplus E_{\infty}$ (for reviews see [25] [26]). Here the sub-bundle is defined by the kernel $E_{x}$ of the linear map $R_{x}$ (2.1). The ADHM equation (2.2) ensures that the connection obtained in this way is anti-self-dual (ASD).

The non-degeneracy condition means the kernel is precisely $n$-dimensional so that we have a rank $n$ non-trivial bundle $E$ (with fibre $E_{x}$ ) over $\mathbb{R}^{4}$.

1 The $n$ dimensional space $E_{\infty}$ becomes the fiber of $E$ at infinity. In the approach of Nahm $E_{\infty}$ and the associated map $P$ originated from the boundary condition at infinity of the adjoint Dirac equation in the instanton background. Sometimes the term $P$ is referred to as the source term which spoils the anti-self-duality of the $T_{i}$ matrices G[5]. The source term arises when we are dealing with non-compact manifold like $\mathbb{R}^{4}$. 
It is convenient to introduce the complex variables;

$$
\tau_{1}=T_{1}+i T_{2}, \quad \tau_{2}=T_{3}+i T_{4}
$$

We also decompose $S^{+}$in two pieces such that

$$
P=a^{*} \otimes<1>+b \otimes<\theta>
$$

where $a: \mathcal{H} \rightarrow E_{\infty}, b: E_{\infty} \rightarrow \mathcal{H}$. Now the ADHM equation becomes

$$
\begin{aligned}
{\left[\tau_{1}, \tau_{2}\right]+b a } & =0, \\
{\left[\tau_{1}, \tau_{1}^{*}\right]+\left[\tau_{2}, \tau_{2}^{*}\right]+b b^{*}-a^{*} a } & =0,
\end{aligned}
$$

where $\tau_{1}, \tau_{2}$ are $k \times k$ matrices, $b$ and $a$ are $k \times n$ and $n \times k$ matrices. The ADHM data can be expressed by the following figure [27];

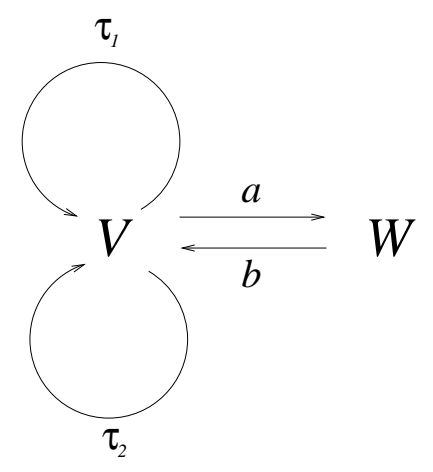

Fig. 1. The ADHM data for $k S U(n)$ instantons: $V=\mathbb{C}^{k}, W=\mathbb{C}^{n}, \tau_{1}, \tau_{2} \in \operatorname{End}(V)$, $a \in \operatorname{Hom}(V, W)$ and $b \in \operatorname{Hom}(W, V)$.

\subsection{The Approach of Donaldson}

Donaldson reformulated the ADHM construction in terms of geometric invariant theory [4]. Let $E$ be an $S U(n)$ bundle over $\mathbb{C P}^{2}$ and consider the moduli space of ASD connections on it. Donaldson identified the instanton moduli space with the moduli space of stable holomorphic $S L(n, \mathbb{C})$ bundles $\mathcal{E}$ which are topologically equivalent to $E\left[30\right.$. Now $\mathbb{R}^{4}$ can be naturally regarded as $\mathbb{C}^{2}$ by picking a complex structure $I$ with $I^{2}=-1$. Donaldson regarded the flat metric on $\mathbb{C}^{2}$ as a metric on $\mathbb{C P}^{2}$ singular along a (complex) line $\ell_{\infty}$ at infinity. Then an instanton on $\mathbb{R}^{4}$ can be identified with an equivalence class of stable holomorphic bundles on $\mathbb{C P}^{2}$ which is trivial precisely along the line $\ell_{\infty}$. So the remaining task is to find such a stable bundle.

This can be accomplished by introducing a monad. Consider homogeneous coordinates $[X]=(x, y, z)$ for $\mathbb{C P}^{2}$ with $z=0$ defining the line $\ell_{\infty}$ at infinite. Let $H, K, L$ be complex 
vector spaces of dimensions $k, 2 k+n, k$, respectively. A monad is linear maps for each $X \in \mathbb{C}^{3}$

$$
H \stackrel{A_{X}}{\longrightarrow} K \stackrel{B_{X}}{\longrightarrow} L
$$

depending linearly on $X$ :

$$
A_{X}=A_{x} x+A_{y} y+A_{z} z, \quad B_{X}=B_{x} x+B_{y} y+B_{z} z,
$$

such that $B_{X} A_{X}: H \rightarrow L$ is zero. This gives a set of six equations. A monad is nondegenerated if for each non-zero $X, A_{X}$ is injective and $B_{X}$ is surjective. This corresponds to the non-degeneracy condition (Sect. 2.1. (iii)) Then a monad defines a complex and a holomorphic vector bundle over $\mathbb{C P}^{2}$ whose fiber at the point $X$ is the vector space $\operatorname{Ker} B_{X} / \operatorname{Im} A_{X}$. The bundles trivial on a fixed line, say $z=0$, can be constructed by purely algebraic considerations. The five conditions

$$
B_{x} A_{x}=B_{y} A_{y}=0, \quad B_{x} A_{y}+B_{y} A_{x}=B_{z} A_{y}+B_{y} A_{z}=B_{z} A_{x}+B_{x} A_{z}=0
$$

can be solved as follows:

$$
B_{z}=\left(\begin{array}{lll}
-\tau_{2} & \tau_{1} & b
\end{array}\right), \quad A_{z}=\left(\begin{array}{c}
\tau_{1} \\
\tau_{2} \\
a
\end{array}\right),
$$

where $\tau_{1}, \tau_{2}$ are $k \times k$ matrices and $b$ and $a$ are $k \times n$ and $n \times k$ matrices. We have a remaining condition:

$$
B_{x} A_{x}=0 \Longrightarrow\left[\tau_{1}, \tau_{2}\right]+b a=0 .
$$

This is the first equation of ADHM in (2.6).

Let $\mathcal{W}_{k, n}$ be the space of quadruples $\left(\tau_{1}, \tau_{2}, a, b\right)$. The space $\overline{\mathcal{W}}_{k, n}^{1,1}$ of monads is defined by (2.11). Let $\mathcal{W}_{k, n}^{1,1}$ be the space of non-degenerated monads. The space $\mathcal{W}_{k, n}^{1,1}$ can be identified with the space $\mathcal{A}_{n, k}^{1,1}$ of framed holomorphic connections of a $S U(n)$ bundle with instanton number $k$. The non-degeneracy condition is identical to the stability of holomorphic vector bundles. On the space of quadruples $\left(\tau_{1}, \tau_{2}, a, b\right)$, we have the natural $G L(k, \mathbb{C})$ action;

$$
\left(\tau_{1}, \tau_{2}, a, b\right) \rightarrow\left(p \tau_{1} p^{-1}, p \tau_{2} p^{-1}, a p^{-1}, p b\right), \quad p \in G L(k, \mathbb{C}) .
$$

A fundamental theorem of Donaldson says that there is one-to-one correspondence between the quotient space $\hat{\mathcal{M}}_{k, n}=\mathcal{W}_{k, n}^{1,1} / G L(k, \mathbb{C})$ and the quotient space $\left(\mathcal{A}^{1,1}\right)_{n, k}^{s} / \mathcal{G}^{\mathbb{C}}$ where $\mathcal{G}^{\mathbb{C}}$

2 A connection $A$ is called framed if it tends to a pure gauge at infinity together with a trivialization at infinity. Throughout this paper, we will always consider the framed connections. 
is the complexification of the infinite-dimensional group $\mathcal{G}$ of the gauge transformation. The latter space can be identified with the moduli space $\mathcal{M}_{n, k}$ of framed ASD connections of gauge group $S U(n)$ with the instanton number $k$. To get the moduli space of unframed instantons, we need to consider $S L(n, \mathbb{C})$ action together with $(2.12)$ :

$$
\left(\tau_{1}, \tau_{2}, a, b\right) \rightarrow\left(p \tau_{1} p^{-1}, p \tau_{2} p^{-1}, q a p^{-1}, p b q^{-1}\right), \quad p \in G L(k, \mathbb{C}), \quad q \in S L(n, \mathbb{C})
$$

The remaining step is to identify the equivalence class of stable orbits of the natural $G L(k, \mathbb{C})$ action (the GIT quotient). This can be done by using the relation between GIT quotient and symplectic quotients. We have a natural Hamiltonian action $U(k)$ on the quadruples $\left(\tau_{1}, \tau_{2}, a, b\right)$ satisfying (2.11) with momentum map (Hamiltonian) $\mu$ given by

$$
\mu_{1}\left(\tau_{1}, \tau_{2}, a, b\right)=\left[\tau_{1}, \tau_{1}^{*}\right]+\left[\tau_{2}, \tau_{2}^{*}\right]+b b^{*}-a^{*} a
$$

This is the second equation of ADHM in (2.6). Now we can form the symplectic quotient $\left.\left(\mu^{-1}(0) \cap \mathcal{W}_{k, n}^{1,1}\right) / U(k)\right)$, which is identical to the GIT quotient $\hat{\mathcal{M}}_{k, n}$. The ADHM theorem says it is diffeomorphic to the moduli space $\mathcal{M}_{n, k}$ of framed instantons. There is a stronger theorem which says that the above two spaces are isometric as hyperKähler manifolds [8]. One may also view the two equations (2.11) and (2.14) as the Hyperkähler momentum maps associated with the hyperkähler structure $I, J, K$ of $\mathbb{R}^{4}$;

$$
\left[\tau_{1}, \tau_{2}\right]+b a=\mu_{2}+i \mu_{3} \equiv \mu_{\mathbb{C}}, \quad\left[\tau_{1}, \tau_{1}^{*}\right]+\left[\tau_{2}, \tau_{2}^{*}\right]+b b^{*}-a^{*} a=\mu_{1} \equiv \mu_{\mathbb{R}}
$$

The non-degeneracy condition corresponds to the stability. If we remove the nondegeneracy condition the quotient space will contain semi-stable orbits. This gives a natural completion $\hat{\mathcal{M}}_{k, r}$ of the moduli space $\hat{\mathcal{M}}_{k, r}$. This property will be important in this paper. If one of the elements of the quadruples is not stable, $\tau_{1}$ and $\tau_{2}$ can be block diagonalized

$$
\begin{aligned}
\tau_{1} & =\left(\begin{array}{cc}
\tau_{1}^{\prime} & 0 \\
0 & \operatorname{diag}\left(\xi_{1}^{1}, \ldots, \xi_{1}^{\ell}\right)
\end{array}\right), \\
\tau_{2} & =\left(\begin{array}{cc}
\tau_{2}^{\prime} & 0 \\
0 & \operatorname{diag}\left(\xi_{2}^{1}, \ldots, \xi_{2}^{\ell}\right)
\end{array}\right), \\
b & =\left(\begin{array}{ll}
b^{\prime} & 0
\end{array}\right), \quad a=\left(\begin{array}{c}
a^{\prime} \\
0
\end{array}\right)
\end{aligned}
$$

where $\tau_{1}^{\prime}, \tau_{2}^{\prime}$ are $(k-\ell) \times(k-\ell)$ matrices, $b^{\prime}$ and $a^{\prime}$ are $(k-\ell) \times n$ and $n \times(k-\ell)$ matrices. Then the equation describes $S U(n)$ instantons of the instanton number $(k-\ell)$ together with $\ell$ point-like instantons. One interprets $\left(\xi_{1}^{i}, \xi_{2}^{i}\right)$ as the complex coordinates of the position of the $i$-th point-like instanton. So one gets

$$
\hat{\mathcal{M}}_{k, n}=\hat{\mathcal{M}}_{k, n} \cup \hat{\mathcal{M}}_{k-1, n} \times \mathbb{C}^{2} \cup \ldots \cup \hat{\mathcal{M}}_{k-\ell, n} \times S^{k-\ell}\left(\mathbb{C}^{2}\right) \ldots \cup S^{k}\left(\mathbb{C}^{2}\right),
$$


where $S^{\ell}(X)$ denotes the $\ell$-th symmetric product of $X$ - the configuration space of $\ell$ non-interacting bosonic particles on $X$. Maciocia showed that this is isometric to the Donaldson-Uhlenbeck compactification

$$
\overline{\mathcal{M}}_{n, k}=\bigcup_{\ell=0}^{k} \mathcal{M}_{n, k-\ell} \times S^{\ell}\left(\mathbb{C}^{2}\right)
$$

of $\mathcal{M}_{n, k}$ [8]. Clearly the space $\overline{\mathcal{M}}_{k, n}$ contains singularities because of the $S^{\ell}\left(\mathbb{C}^{2}\right)$.

Through out this paper, we will not impose the non-degeneracy condition unless specified. For example,

$$
\overline{\hat{\mathcal{M}}}_{k, n}=\left(\mu_{\mathbb{C}}^{-1}(0) \cap \mu_{\mathbb{R}}^{-1}(0)\right) / U(k) .
$$

Because of the isometry between the two moduli spaces $\overline{\mathcal{M}}_{k, n}$ and $\overline{\mathcal{M}}_{n, k}$, we will often not distinguish them.

\section{Extension}

\subsection{An Extension of Monads}

Now we consider a simple modification of the monad construction in the previous subsection. To begin with we consider monads for $S U(2 N)$ instantons with instanton number $N$. We have three complex vector spaces $H, K, L$ of dimensions $N, 4 N, N$, respectively. The five conditions (2.9) can be solved via

$$
\begin{aligned}
& B_{x}=\left(\begin{array}{llll}
0 & 1 & 0 & 0
\end{array}\right), \quad B_{y}=\left(\begin{array}{llll}
-1 & 0 & 0 & 0
\end{array}\right), \quad B_{z}=\left(\begin{array}{llll}
-t_{2} & t_{1} & -t_{4} & t_{3}
\end{array}\right), \\
& A_{x}=\left(\begin{array}{l}
1 \\
0 \\
0 \\
0
\end{array}\right), \quad A_{y}=\left(\begin{array}{l}
0 \\
1 \\
0 \\
0
\end{array}\right), \quad A_{z}=\left(\begin{array}{c}
t_{1} \\
t_{2} \\
t_{3} \\
t_{4}
\end{array}\right) \text {, }
\end{aligned}
$$

where $t_{1}, t_{2}, t_{3}, t_{4}$ are $N \times N$ matrices. Note that $B=A^{t}(J \oplus J)$ where $J=\left(\begin{array}{cc}0 & 1 \\ -1 & 0\end{array}\right)$, in the notation of block matrix. The remaining condition $B_{x} A_{x}=0$ is identical to

$$
B_{x} A_{x}=0 \Longrightarrow\left[t_{1}, t_{2}\right]+\left[t_{3}, t_{4}\right]=0
$$

We may put $\left(\begin{array}{ll}-t_{4} & t_{3}\end{array}\right)$ into a $N \times 2 N$ matrix, which we call $b$. In the standard interpretation, we are considering a special subset of monads for $S U(2 N)$ and $N$ instantons. In (3.1), we fixed a preferred basis for the $N \times 2 N$ matrix $b$ and $2 N \times N$ matrix $a$;

$$
b=a^{t} J \Longleftrightarrow\left(\begin{array}{ll}
-t_{4} & t_{3}
\end{array}\right)=\left(\begin{array}{ll}
t_{3} & t_{4}
\end{array}\right)\left(\begin{array}{cc}
0 & 1 \\
-1 & 0
\end{array}\right) .
$$


Our preferred choice of the basis is invariant under the $G L(N, \mathbb{C}$ ) action (for example, the left action on $b$ ). In contrast to the usual $S U(2 N)$ case, our basis is not invariant under the $S L(2 N, \mathbb{C}$ ) action ( for example, the right action on $b$ ) but invariant under $G L(N, \mathbb{C}) \oplus G L(N, \mathbb{C})$ action. Now we formulate the following $G L(N, \mathbb{C})$ action on the quadruples $\left(t_{1}, t_{2}, t_{3}, t_{4}\right)$

$$
\left(t_{1}, t_{2}, t_{3}, t_{4}\right) \rightarrow\left(p t_{1} p^{-1}, p t_{2} p^{-1}, p t_{3} p^{-1}, p t_{4} p^{-1}\right), \quad p \in G L(N, \mathbb{C}) .
$$

This should be compared with (2.12) and (2.13). Here we put $\left(t_{1}, t_{2}\right)$ and $\left(t_{3}, t_{4}\right)$ on equal footing.

Let $\mathfrak{A}_{N}$ denote the space of quadruples $\left(t_{1}, t_{2}, t_{3}, t_{4}\right)$ of $N \times N$ matrices. The space $\overline{\mathfrak{A}}_{N}^{1,1}$ of monads is subset of $\mathfrak{A}_{N}$ satisfying (3.2). We write $\mathfrak{A}_{N}^{1,1}$ for the non-degenerated subset of $\overline{\mathfrak{A}}_{N}^{1,1}$

The remaining step is to identify the equivalence class of stable orbits of the $G L(N, \mathbb{C})$ action (3.4) (the GIT quotient). We have a natural adjoint action $U(N)$ on the quadruples $\left(t_{1}, t_{2}, t_{3}, t_{4}\right)$ preserving the obvious norm. The momentum map (Hamiltonian) $\tilde{\mu}_{1}$ is given by

$$
\tilde{\mu}_{1}\left(t_{1}, t_{2}, t_{3}, t_{4}\right)=\left[t_{1}, t_{1}^{*}\right]+\left[t_{2}, t_{2}^{*}\right]+\left[t_{3}, t_{3}^{*}\right]+\left[t_{4}, t_{4}^{*}\right] .
$$

This form of the momentum map is follows from (3.4). Then we have the symplectic quotient, with the non-degeneracy condition

$$
\mathfrak{M}_{N}=\left(\mathfrak{A}_{N}^{1,1} \cap \tilde{\mu}_{1}^{-1}(0)\right) / U(N)
$$

which is isomorphic to the GIT quotient, $\mathfrak{A}_{N}^{1,1} / / G L(N, \mathbb{C})$. We will call the above space the extended moduli space of framed instantons.

Finally, we note that the complex equation (3.2) together with the real equation (3.5) can be written in terms of hyperkähler momentum maps:

$$
\begin{aligned}
\tilde{\mu}_{\mathbb{C}} & \equiv\left[t_{1}, t_{2}\right]+\left[t_{3}, t_{4}\right]=0, \\
\tilde{\mu}_{\mathbb{R}} & \equiv\left[t_{1}, t_{1}^{*}\right]+\left[t_{2}, t_{2}^{*}\right]+\left[t_{3}, t_{3}^{*}\right]+\left[t_{4}, t_{4}^{*}\right]=0 .
\end{aligned}
$$

After removing the non-degeneracy condition we can write (3.6) as

$$
\overline{\mathfrak{M}}_{N}=\left(\tilde{\mu}_{\mathbb{C}}^{-1}(0) \cap \tilde{\mu}_{\mathbb{R}}^{-1}(0)\right) / U(N) .
$$

\subsection{Dolbeault Equivariant Cohomology and $S^{1}$ Actions}

Recall the natural adjoint $U(N)$ action on the quadruples $\left(t_{1}, t_{2}, t_{3}, t_{4}\right)$ :

$$
\left(t_{1}, t_{2}, t_{3}, t_{4}\right) \rightarrow\left(g t_{1} g^{-1}, g t_{2} g^{-1}, g t_{3} g^{-1}, g t_{4} g^{-1}\right), \quad g \in U(N)
$$


The infinitesimal variation of this action is given by

$$
\delta t_{i}=\left[\delta g, t_{i}\right], \quad \delta t_{i}^{*}=-\left[\delta g, t_{i}^{*}\right]
$$

for $i=1,2,3,4$.

In the space of the quadruples, we have an obvious inner products and the Kähler potential $\mathfrak{k}$ is given by

$$
\mathfrak{k}=-\frac{1}{2} \operatorname{tr}\left(\left|t_{1}\right|^{2}+\left|t_{2}\right|^{2}+\left|t_{3}\right|^{2}+\left|t_{4}\right|^{2}\right)
$$

In addition to the adjoint $U(N)$ actions, we also have natural $S^{1}$-actions on the quadruples preserving the natural norm. Such an action may be called a hyper-Hamiltonian action if it preserves the hyperkähler momentum maps (3.7). We have the following hyperHamiltonian $S^{1} \times S^{1}$ actions

$$
\left(t_{1}, t_{2}, t_{3}, t_{4}\right) \rightarrow\left(e^{-i m^{\prime} \varphi^{\prime}} t_{1}, e^{+i m^{\prime} \varphi^{\prime}} t_{2}, e^{+i m^{\prime \prime} \varphi^{\prime \prime}} t_{3}, e^{-i m^{\prime \prime} \varphi^{\prime \prime}} t_{4}\right),
$$

where $\varphi^{\prime}$ and $\varphi^{\prime \prime}$ denote the generators of $S_{m^{\prime}}^{1}$ actions on $\left(t_{1}, t_{2}, t_{1}^{*}, t_{2}^{*}\right)$ and of $S_{m^{\prime \prime}}^{1}$ action on $\left(t_{3}, t_{4}, t_{3}^{*}, t_{4}^{*}\right)$, respectively. Clearly the two $S^{1}$ actions are disjoint at least for the equation (3.7). Now the combined action of $U(N) \times S_{m^{\prime}}^{1} \times S_{m^{\prime \prime}}^{1}$ can be summarized as

$$
\begin{array}{ll}
\delta t_{1}=+i m^{\prime} t_{1}+\left[\delta g, t_{1}\right], & \delta t_{3}=+i m^{\prime \prime} t_{3}+\left[\delta g, t_{3}\right], \\
\delta t_{2}=-i m^{\prime} t_{2}+\left[\delta g, t_{2}\right], & \delta t_{4}=-i m^{\prime \prime} t_{4}+\left[\delta g, t_{4}\right] .
\end{array}
$$

It is convenient to formulate a Dolbeault version of equivariant cohomology of the combined action of $U(N) \times S_{m^{\prime}}^{1} \times S_{m^{\prime \prime}}^{1}$. Using field theory language 3 , we define the basic supersymmetry algebra generated by two global supercharge $\mathbf{s}$ and $\overline{\mathbf{s}}$

$$
\begin{array}{ll}
\mathbf{s} t_{i}=\psi_{i}, & \mathbf{s} \psi_{i}=0, \\
\overline{\mathbf{s}} t_{i}=0, & \overline{\mathbf{s}} \psi_{i}=-i\left[t_{0}, t_{i}\right]-i m^{\prime} t_{i}\left(\delta_{1 i}-\delta_{2 i}\right)-i m^{\prime \prime} t_{i}\left(\delta_{3 i}-\delta_{4 i}\right), \\
\mathbf{s} t_{i}^{*}=0, & \mathbf{s} \psi_{i}^{*}=-i\left[t_{0}, t_{i}^{*}\right]+i m^{\prime} t_{i}^{*}\left(\delta_{1 i}-\delta_{2 i}\right)+i m^{\prime \prime} t_{i}^{*}\left(\delta_{3 i}-\delta_{4 i}\right), \\
\overline{\mathbf{s}} t_{i}^{*}=\psi_{i}^{*}, & \overline{\mathbf{s}} \psi_{i}^{*}=0,
\end{array}
$$

where $i=1,2,3,4, t_{0}=t_{0}^{*}$ is a $N \times N$ matrix, $m^{\prime}, m^{\prime \prime}$ are real positive numbers and $\psi_{i}$ and $\psi_{i}^{*}$ are $N \times N$ matrix with anti-commuting matrix elements. The algebra satisfies $\mathbf{s}^{2}=\overline{\mathbf{s}}^{2}=0$ and

$$
\begin{aligned}
& \{\mathbf{s}, \overline{\mathbf{s}}\} t_{i}=-i\left[t_{0}, t_{i}\right]-i m^{\prime} t_{i}\left(\delta_{1 i}-\delta_{2 i}\right)-i m^{\prime \prime} t_{i}\left(\delta_{3 i}-\delta_{4 i}\right), \\
& \{\mathbf{s}, \overline{\mathbf{s}}\} t_{i}^{*}=-i\left[t_{0}, t_{i}^{*}\right]+i m^{\prime} t_{i}^{*}\left(\delta_{1 i}-\delta_{2 i}\right)+i m^{\prime \prime} t_{i}^{*}\left(\delta_{3 i}-\delta_{4 i}\right),
\end{aligned}
$$

3 This is the obvious cousin of the field theoretical counterparts in [31]. 
which show that the commutator $\{\mathbf{s}, \overline{\mathbf{s}}\}$ generate the infinitesimal $U(N) \times S_{m^{\prime}}^{1} \times S_{m^{\prime \prime}}^{1}$ symmetry as given in (3.13). This is the equivariant extension of the usual Dolbeault cohomology.

We compute the equivariant extension of the Kähler form;

$$
-\frac{i}{2}(\mathbf{s} \overline{\mathbf{s}}-\overline{\mathbf{s}} \mathbf{s}) \mathfrak{k}=-\frac{1}{2} \operatorname{tr}\left(t_{0} \mu_{\mathbb{R}}+m^{\prime}\left(t_{1} t_{1}^{*}-t_{2} t_{2}^{*}\right)+m^{\prime \prime}\left(t_{3} t_{3}^{*}-t_{4} t_{4}^{*}\right)-i \psi_{i} \psi_{i}^{*}\right),
$$

where the repeated indices are summed over. One can identify the first 3 terms with the momentum maps of $U(N), S_{m^{\prime}}^{1}$ and $S_{m^{\prime \prime}}^{1}$ actions, respectively. The last term $\frac{i}{2} \operatorname{tr}\left(\psi_{i} \psi_{i}^{*}\right)$ can be identified with the Kähler form.

There are other $S^{1}$ actions preserving either $\tilde{\mu}_{\mathbb{C}}$ or $\tilde{\mu}_{\mathbb{R}}$ which will be considered later.

\section{3. $S_{m^{\prime \prime}}^{1}$ Action and Fixed Points}

The two sets of matrices $\left(t_{1}, t_{2}\right)$ and $\left(t_{3}, t_{4}\right)$ are on equal footing in our formulation. The $4 N \times N$ matrices can be viewed as 4 endomorphisms of $V=\mathbb{C}^{N}$, i.e. $t_{1}, t_{2}, t_{3}, t_{4} \in$ $\operatorname{End}\left(\mathbb{C}^{N}\right)$, with $G L(N, \mathbb{C})$ action.

To relate the ADHM constructions and Sect. 5.1, we will consider the $S_{m^{\prime \prime}}^{1}$ action only and its fixed points. We set $m^{\prime}=0$ and $m^{\prime \prime}=m$. The momentum map of $S_{m}^{1}$ action is given by $\frac{m}{2} \operatorname{tr}\left(t_{3} t_{3}^{*}-t_{4} t_{4}^{*}\right)$. If we write an elemet of $U(N)$ as $e^{i t_{0}^{a} T^{a}}$, where $T^{a}$ denotes the generator of Lie algebra of $U(N)$ in the adjoint representation, we have the following infinitesimal form of the combined action of $U(N) \times S_{m}^{1}$

$$
\left\{\begin{array} { l } 
{ \delta t _ { 1 } = i [ t _ { 0 } , t _ { 1 } ] , } \\
{ \delta t _ { 2 } = i [ t _ { 0 } , t _ { 2 } ] , } \\
{ \delta t _ { 1 } ^ { * } = i [ t _ { 0 } , t _ { 1 } ^ { * } ] , } \\
{ \delta t _ { 2 } = i [ t _ { 0 } , t _ { 2 } ^ { * } ] , }
\end{array} \quad \left\{\begin{array}{l}
\delta t_{3}=+i m t_{3}+i\left[t_{0}, t_{3}\right], \\
\delta t_{4}=-i m t_{4}+i\left[t_{0}, t_{4}\right], \\
\delta t_{3}^{*}=-i m t_{3}^{*}+i\left[t_{0}, t_{3}^{*}\right] \\
\delta t_{4}^{*}=+i m t_{4}^{*}+i\left[t_{0}, t_{4}^{*}\right] .
\end{array}\right.\right.
$$

Now we examine the various fixed point of the $S^{1}$ action. The most obvious one occurs when $t_{3}=t_{4}=0$. There are other kinds of fixed points if and only if the $U(N)$ symmetry breaks down such that the unbroken parts the symmetry can undo the $S_{m}^{1}$ action. So the fixed points of $S_{m}^{1}$ action are identical to the fixed point of the infinitesimal action of $U(N) \times S_{m}^{1}$ described in (3.17), together with the symmetry breaking pattern. Note that the above procedure is equivalent to finding the fixed points of the global supersymmetry $\overline{\mathbf{s}} \psi_{i}=\mathbf{s} \psi_{i}^{*}=0$.

For example the fixed point equation $\delta t_{3}=0$ reduces to solving an eigenvalue problem of operator $\left[t_{0}, \quad\right]$ acting on $t_{3}$ with eigenvalue $-m,\left[t_{0}, t_{3}\right]=m t_{3}$. Thus $t_{0}$ should be diagonalized, $t_{0}=U \phi U^{\dagger}$;

$$
\phi=\operatorname{diag}\left(s_{1}, \ldots, s_{N}\right)
$$


We will order the diagonal components, using the Weyl group, as $s_{1} \geq \ldots \geq s_{N}$. Let $\left\{k_{\ell}\right\}, \ell=1, \ldots, n_{p}$ be a partition of $N$ by positive integers $k_{\ell}, N=\sum_{\ell=1}^{n_{p}} k_{\ell}$. To a given partitions, we associate

$$
\phi=\operatorname{bdiag}\left(\alpha_{1} I_{k_{1}}, \ldots, \alpha_{n_{p}} I_{k_{n_{p}}}\right),
$$

where $I_{k_{\ell}}$ denotes $k_{\ell} \times k_{\ell}$ unit matrix and $\alpha_{1}>\ldots>\alpha_{n_{p}}$. If, for example, $n_{p}$ is identical to $N$, we have $k_{\ell}=1$ for all $\ell=1, \ldots, N$ and the all eigenvalues $\alpha_{\ell}$ of $\phi$ in (3.18) are distinct. The eignenvalues of $\phi$ determine the symmetry breaking pattern. In other words we have the eigenspace decomposition:

$$
V=\oplus_{\ell} V_{\ell}, \quad \text { with } \quad N=\sum_{\ell=1}^{n_{p}} k_{\ell}, \quad V_{\ell}=\mathbb{C}^{k_{\ell}}
$$

For a fixed partition $\left\{k_{\ell}\right\}$ with $\ell=1, \ldots, n_{p}$, the fixed points of the $S^{1}$ action depending on (3.19) are easily determined. The second set of equations in (3.17) implies $t_{3}$ becomes block upper trianglular, provided that $m>0$. Then $t_{3}$ has $n_{p} \times n_{p}$ blocks and $\left(\ell, \ell^{\prime}\right)$-th block denoted by $\sigma_{\ell, \ell^{\prime}}$ is a $k_{\ell} \times k_{\ell^{\prime}}$ matrix with $\sigma_{\ell, \ell^{\prime}}=0$ for $\ell \geq \ell^{\prime}$. It is also easy to see that not all of the $\sigma_{\ell, \ell^{\prime}}$ with $\ell>\ell^{\prime}$ are non-vanishing. In general a $k_{\ell} \times k_{\ell^{\prime}}$ matrix $\sigma_{\ell, \ell^{\prime}}$ vanishes if

$$
\alpha_{\ell}-\alpha_{\ell^{\prime}} \neq m
$$

Otherwise there is no restriction. Note that we have arranged that $\alpha_{\ell}>\alpha_{\ell^{\prime}}$ if $\ell>\ell^{\prime}$ and $m$ is a positive number. Similarly, $t_{4}$ has $n_{p} \times n_{p}$ blocks and the $\left(\ell, \ell^{\prime}\right)$-th block denoted by $\pi_{\ell, \ell^{\prime}}$ is a $k_{\ell} \times k_{\ell^{\prime}}$ matrix with $\pi_{\ell, \ell^{\prime}}=0$ for $\ell \leq \ell^{\prime}$. When the condition $\alpha_{\ell}-\alpha_{\ell^{\prime}}=m$ is satisfied, the matrix $\pi_{\ell^{\prime}, \ell}$ has no restriction. On the other hand, if $\alpha_{\ell}-\alpha_{\ell^{\prime}} \neq m$ the matrix $\pi_{\ell^{\prime}, \ell}$ is identically zero. The first set of equations in (3.17) implies $t_{1}$ and $t_{2}$ become block diagonal with $n_{p}$ blocks. We will denote the block elements of $t_{1}$ and $t_{2}$ by $z_{\ell}$ and $w_{\ell}$, repectively; both are $k_{\ell} \times k_{\ell}$ matrices.

The above discussions can be summarized as follows. Under the eigenspace decompositions (3.20), the original endomorphism of $V$ is decomposed into $\operatorname{End}\left(V_{\ell}\right)$ and $\operatorname{Hom}\left(V_{\ell}, V_{\ell^{\prime}}\right)$ with $\ell, \ell^{\prime} \in 1, \ldots, n_{p}$. Then we have identifications: $z_{\ell}, w_{\ell} \in \operatorname{End}\left(V_{\ell}\right), \sigma_{\ell, \ell^{\prime}} \in \operatorname{Hom}\left(V_{\ell}, V_{\ell^{\prime}}\right)$ and $\pi_{\ell^{\prime}, \ell} \in \operatorname{Hom}\left(V_{\ell^{\prime}}, V_{\ell}\right)$ with $\ell<\ell^{\prime}$.

\subsubsection{Examples}

Now we consider some examples of fixed points.

\section{Example (1)}

Consider all eigenvalues in (3.18) are the same. The only solution of (3.17) is $t_{3}=t_{4}=$ 0 . There are no restrictions to $t_{1}$ and $t_{2}$. The equation (3.7) reduces to

$$
\left[t_{1}, t_{2}\right]=0, \quad\left[t_{1}, t_{1}^{*}\right]+\left[t_{2}, t_{2}^{*}\right]=0 .
$$


The original $U(N)$ symmetry is unbroken. One obvious family of solutions of above for $t_{1}, t_{2}$ are diagonalized matrices;

$$
t_{1}=\operatorname{diag}\left(z^{1}, \ldots, z^{N}\right), \quad t_{2}=\operatorname{diag}\left(w^{1}, \ldots, w^{N}\right)
$$

One can regard the eigenvalues as the positions of $N$ unordered points in $\mathbb{C}^{2}$. So the fixed point locus is $S^{N}\left(\mathbb{C}^{2}\right)$. Note that $\frac{1}{N}\left(\operatorname{tr} t_{1}, \operatorname{tr} t_{2}\right)$ can be interpreted as the center of mass coordinates in $\mathbb{C}^{2}$. This is the ADHM description for $N$ point-like instantons.

Example (2)

We consider a partition $N=k+n$ and $\phi=\operatorname{bdiag}\left(\alpha I_{k},(\alpha-m) I_{n}\right)$ leading to the following eigen space decomposition;

$$
V=V_{1} \oplus V_{2}, \quad V_{1}=\mathbb{C}^{k}, \quad V_{2}=\mathbb{C}^{n},
$$

The original $U(N)(G L(N, \mathbb{C}))$ symmetry is broken to $U(k) \times U(n)(G L(k, \mathbb{C}) \times G L(n, \mathbb{C}))$. We have

$$
t_{1}=\left(\begin{array}{cc}
z_{1} & 0 \\
0 & z_{2}
\end{array}\right), \quad t_{2}=\left(\begin{array}{cc}
w_{1} & 0 \\
0 & w_{2}
\end{array}\right), \quad t_{3}=\left(\begin{array}{cc}
0 & \sigma_{1,2} \\
0 & 0
\end{array}\right), \quad t_{4}=\left(\begin{array}{cc}
0 & 0 \\
\pi_{2,1} & 0
\end{array}\right),
$$

where $\sigma_{1,2}$ is $k \times n$ and $\pi_{2,1}$ is $n \times k$ matrices. In other words, we have $z_{1}, w_{1} \in \operatorname{End}\left(V_{1}\right)$, $z_{2}, w_{2} \in \operatorname{End}\left(V_{2}\right), \sigma_{1,2} \in \operatorname{Hom}\left(V_{2}, V_{1}\right)$ and $\pi_{2,1} \in \operatorname{Hom}\left(V_{1}, V_{2}\right)$. We have

$$
\left\{\begin{array} { l } 
{ [ z _ { 1 } , w _ { 1 } ] + \sigma \pi = 0 , } \\
{ [ z _ { 1 } , z _ { 1 } ^ { * } ] + [ w _ { 1 } , w _ { 1 } ^ { * } ] + \sigma \sigma ^ { * } - \pi ^ { * } \pi = 0 , }
\end{array} \quad \left\{\begin{array}{l}
{\left[z_{2}, w_{2}\right]-\pi \sigma=0,} \\
{\left[z_{2}, z_{2}^{*}\right]+\left[w_{2}, w_{2}^{*}\right]+\pi \pi^{*}-\sigma^{*} \sigma=0,}
\end{array}\right.\right.
$$

Note that the first and the second sets of equations describe $k S U(n)$ and $n S U(k)$ instantons, repectively. Assumming the non-degeneracy condition, the fixed point locus is $\left(\left(\mathcal{M}_{n, k}\right) \times\left(\mathcal{M}_{k, n}\right)\right) / S_{2}$, where $S_{2}$ for $k \neq 0$, is the remnant the Weyl group of $U(N)$.

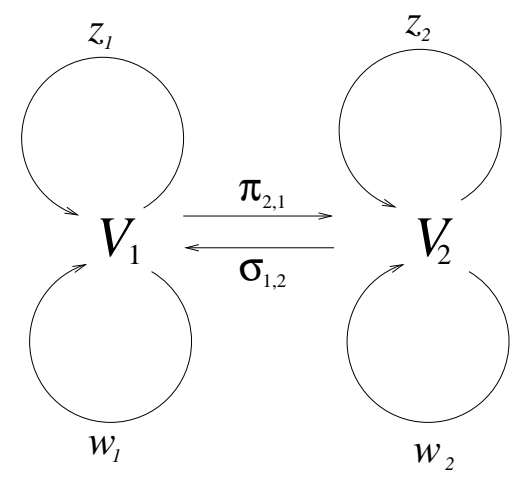

Fig. 2. The example (2): $V=V_{1} \oplus V_{2}$ where $V_{1}=\mathbb{C}^{k}, V_{2}=\mathbb{C}^{n}$ with $N=n+k$, $z_{1}, w_{1} \in \operatorname{End}\left(V_{1}\right), z_{2}, w_{2} \in \operatorname{End}\left(V_{2}\right), \sigma_{1,2} \in \operatorname{Hom}\left(V_{2}, V_{1}\right)$ and $\pi_{2,1} \in \operatorname{Hom}\left(V_{1}, V_{2}\right)$. This is to be 
compared with the usual ADHM description in Fig. 1. In a latter section we will interpret this diagram in terms of $N$ D-instatons confined on two 4-dimensional hyperplanes in 10-dimensions. The endomorphisms and homomorphisms will be interpreted as open strings joining the various D-instantons.

Removing the non-degeneracy condition the first equation in 3.25) has lower strata $\mathcal{M}_{N-k, k-\ell} \times S^{\ell}\left(\mathbb{C}^{2}\right)$ for $\ell=1, \ldots k$. That is $\sigma$ and $\pi$ degenerate to $(k-\ell) \times(N-k)$ and $(N-k) \times(k-\ell)$ matrices, respectively. Then the second equation describe $N-k S U(k-\ell)$ non-degenerated instantons. Then we have $\left(\left(\mathcal{M}_{N-k, k-\ell} \times S^{\ell}\left(\mathbb{C}^{2}\right)\right) \times\left(\mathcal{M}_{k-\ell, N-k}\right)\right) / S_{2}$. Now the $N-k S U(k-\ell)$ instantons can also degenerate to $N-k-\ell^{\prime} S U(k-\ell)$ instantons, for $\ell=1, \ldots, N-k$. Then the first equation describes $k-\ell S U\left(N-k-\ell^{\prime}\right)$ instantons;

$$
\left(\left(\mathcal{M}_{N-k-\ell^{\prime}, k-\ell} \times S^{\ell}\left(\mathbb{C}^{2}\right)\right) \times\left(\mathcal{M}_{k-\ell, N-k-\ell^{\prime}} \times S^{\ell^{\prime}}\left(\mathbb{C}^{2}\right)\right)\right) / S_{2}
$$

Consequently the fixed point locus without the non-degeneracy condition is

$$
\bigcup_{\substack{\ell=0, \ldots, k \\ \ell^{\prime}=0, \ldots, N-k}}\left(\left(\mathcal{M}_{N-k-\ell^{\prime}, k-\ell} \times S^{\ell}\left(\mathbb{C}^{2}\right)\right) \times\left(\mathcal{M}_{k-\ell, N-k-\ell^{\prime}} \times S^{\ell^{\prime}}\left(\mathbb{C}^{2}\right)\right)\right) / S_{2} .
$$

Here we obtain two sets of Yang-Mills instantons correlated with each other in somewhat misterious way. In the later sections, we will intepret our extended monads to describe D-instantons. According to D-instantons picture, the above system describes $k$ and $n$ Dinstantons confined in two parallel 4-dimensional hyperplanes in ten-dimensional space. The strange correlation will be explained by strings. For an observer in a hyperplane, the above system is viewed as

$$
\bigcup_{\substack{\ell=0, \ldots, k \\ \ell^{\prime}=0, \ldots, N-k}}\left(\mathcal{M}_{N-k-\ell^{\prime}, k-\ell} \times S^{\ell}\left(\mathbb{C}^{2}\right)\right)=\bigcup_{\ell^{\prime}=0}^{N-k} \overline{\mathcal{M}}_{N-k-\ell^{\prime}, k-\ell}
$$

We may interpret the above moduli space as a double stratification of the moduli space of $k S U(N-k)$ instantons, one way by $\ell$ number of point-like instantons and the other way by $\ell^{\prime}$ number of flat-factors of $S U(N-k)$ bundles. 1 Then the system described by (3.27) is manifestly reciprocal. The $S_{2}$ action exchanges two $\mathbb{C}^{2}$ and the rank of gauge group and the instanton numbers as well as the two stratifications defined by the flat factors and point-like instantons. Analogous things happen in case of Fourier-Nahm transformation of instantons on a flat 4 -torus [7] [26].

4 A flat factor is the reducible connection $E=E_{0} \oplus L_{f}$, where $L_{f}$ has flat $U(1)$ connections. 
Example (3)

Let $\phi$ is given by (3.19). The maximum non-vanishing number of $\sigma_{\ell, \ell^{\prime}}$ is $n_{p}-1$ when the values $\alpha_{\ell}$ are evenly spaced by $m$, i.e., $\alpha_{1}, \alpha_{1}-m, \alpha_{1}-2 m, \ldots, \alpha_{1}-\left(n_{p}-1\right) m$. Then the non-vanishing elements of $t_{3}$ are $\sigma_{\ell, \ell+1}, \ell=1, \ldots, n_{p}$. Similarly, the non-vanishing elements of $t_{4}$ are $\pi_{\ell, \ell-1}$. If $n_{p}=3$, for example, we have

$$
t_{1}=\left(\begin{array}{ccc}
z_{1} & 0 & 0 \\
0 & z_{2} & 0 \\
0 & 0 & z_{3}
\end{array}\right), \quad t_{3}=\left(\begin{array}{ccc}
0 & \sigma_{1,2} & 0 \\
0 & 0 & \sigma_{2,3} \\
0 & 0 & 0
\end{array}\right), \quad t_{4}=\left(\begin{array}{ccc}
0 & 0 & 0 \\
\pi_{2,1} & 0 & 0 \\
0 & \pi_{3,2} & 0
\end{array}\right)
$$

with the following diagram;

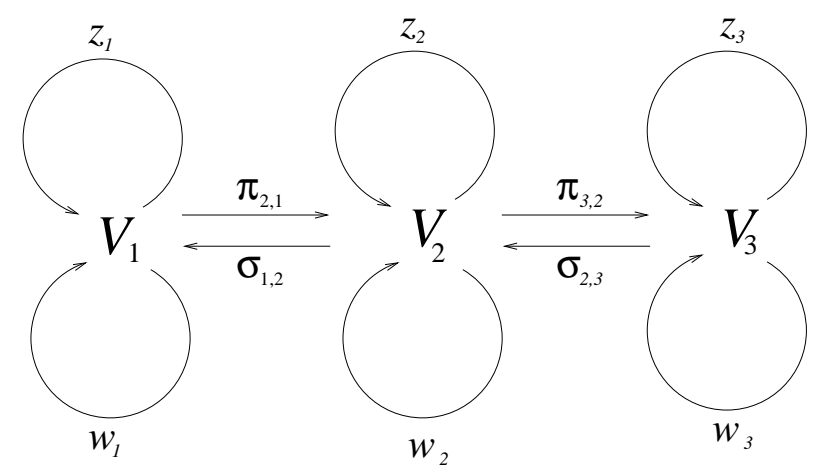

Fig. 3. The eigen space decomposition $V=V_{1} \oplus V_{2} \oplus V_{3}, V_{\ell}=\mathbb{C}^{k_{\ell}}$ with $\sum_{\ell=1}^{3} k_{\ell}=N$.

The equations (3.7) becomes

$$
\begin{aligned}
& {\left[z_{1}, w_{1}\right]+\sigma_{1,2} \pi_{2,1}=0} \\
& {\left[z_{1}, z_{1}^{*}\right]+\left[w_{1}, w_{1}^{*}\right]+\sigma_{1,2} \sigma_{1,2}^{*}-\pi_{2,1}^{*} \pi_{2,1}=0 \text {, }} \\
& {\left[z_{\ell}, w_{\ell}\right]+\sigma_{\ell, \ell+1} \pi_{\ell+1, \ell}-\pi_{\ell, \ell-1} \sigma_{\ell-1, \ell}=0,} \\
& {\left[z_{\ell}, z_{\ell}^{*}\right]+\left[w_{\ell}, w_{\ell}^{*}\right]+\sigma_{\ell, \ell+1} \sigma_{\ell, \ell+1}^{*}-\pi_{\ell+1, \ell}^{*} \pi_{\ell+1, \ell}-\sigma_{\ell-1, \ell}^{*} \sigma_{\ell-1, \ell}+\pi_{\ell, \ell-1} \pi_{\ell, \ell-1}^{*}=0 \text {, }} \\
& {\left[z_{n_{p}}, w_{n_{p}}\right]-\pi_{n_{p}, n_{p}-1} \sigma_{n_{p}-1, p}=0,} \\
& {\left[z_{n_{p}}, z_{n_{p}}^{*}\right]+\left[w_{n_{p}}, w_{n_{p}}^{*}\right]+\sigma_{n_{p}-1, n_{p}}^{*} \sigma_{n_{p}-1, n_{p}}-\pi_{n_{p}, n_{p}-1} \pi_{n_{p}-1, n_{p}}^{*}=0 .}
\end{aligned}
$$

The first two equations of (3.30) are precisely the ADHM equations for $S U\left(k_{2}\right)$ instantons with instanton number $k_{1}$ and last two equations describe $S U\left(k_{n_{p}}\right)$ instantons with instanton numbers $k_{n_{p}-1}$. The two equations in the middle of (3.30) may be identified with the $\mathrm{ADHM}$ equations for the instanton number $k_{\ell}$ with gauge group $S U\left(k_{\ell+1}\right) \times S U\left(k_{\ell-1}\right)$. 
Now the fixed point locus is much more complicated. One can do the similar analysis as the example (2). We only note that the above system has $S_{n_{p}}$ symmetry.

We see that the series of ADHM equations in (3.30) and the corresponding diagram, for example Fig. 3., have certain similarity with ADHM description of instantons on ALE space and the quiver diagram [20][32] [21].

Example (4)

Let $\phi$ is given by (3.19). When $\left|\alpha_{\ell}-\alpha_{\ell^{\prime}}\right| \neq m$ for all $\ell, \ell^{\prime}$, all the matrices $\sigma_{\ell, \ell^{\prime}}$ and $\pi_{\ell, \ell^{\prime}}$ vanish, i.e. $t_{3}=t_{4}=0$. Then the equations (3.7) becomes

$$
\begin{aligned}
{\left[z_{\ell}, w_{\ell}\right] } & =0, \\
{\left[z_{\ell}, z_{\ell}^{*}\right]+\left[w_{\ell}, w_{\ell}^{*}\right] } & =0,
\end{aligned}
$$

where $z_{\ell}$ and $w_{\ell}$ are $k_{\ell} \times k_{\ell}$ matrices. This is the completely degenerated limit of example (3). The system described by (3.31) corresponds to $n_{p}$ paralell 4-dimensional hyperplanes containing $k_{\ell}$ point-like instantons in $\ell$-th hyperplane.

\section{4. $S_{m_{0}}^{1}$ Action on ADHM Data and the Stratification}

In the previous subsection, we recovered the usual ADHM construction by considering fixed points of the natural $S_{m}^{1}$ action. Within a fixed points locus, we still have the $S^{1}$ action. So we will return to the case reviewed in Sect. 2.2 and use the same notation. We had the quadruples $\left(\tau_{1}, \tau_{2}, a, b\right)$, with $G L(k, \mathbb{C})$ action

$$
\left(\tau_{1}, \tau_{2}, a, b\right) \rightarrow\left(p \tau_{1} p^{-1}, p \tau_{2} p^{-1}, a p^{-1}, p b\right), \quad p \in G L(k, \mathbb{C})
$$

We had momentum maps;

$$
\begin{aligned}
& \mu_{\mathbb{C}}=\left[\tau_{1}, \tau_{2}\right]+b a, \\
& \mu_{\mathbb{R}}=\left[\tau_{1}, \tau_{1}^{*}\right]+\left[\tau_{2}, \tau_{2}^{*}\right]+b b^{*}-a^{*} a,
\end{aligned}
$$

and the quotient $\left(\mu_{\mathbb{R}}^{-1}(0) \cap \mu_{\mathbb{C}}^{-1}(0) / U(k)\right)=\overline{\mathcal{M}}_{k, n}$. We consider the following $S_{m_{0}}^{1}$ action on the above quotient space;

$$
\left(\tau_{1}, \tau_{2}, a, b\right) \rightarrow\left(\tau_{1}, \tau_{2}, e^{i m_{0} \varphi_{0}} a, e^{-i m_{0} \varphi_{0}} b\right)
$$

This is analogous to the $S^{1}$ action considered in Sect. 3.2 and preserves both real and complex momentum maps. 
Let $h$ denote a infinitesimal generator of the $U(k)$ acting as (3.32). The combined action of $U(k)$ and $S^{1}$ is given by

$$
\begin{aligned}
\delta \tau_{1} & =i\left[h, \tau_{1}\right], & \delta a & =+i m a-i a h, \\
\delta \tau_{2} & =i\left[h, \tau_{2}\right], & \delta b & =-i m b+i h b .
\end{aligned}
$$

The above transformation law should be compared with (3.17). One now repeat what we did in Sect. 3.1.2. The momentum map of the $S_{m_{0}}^{1}$ is given by $\frac{m_{0}}{2} \operatorname{tr}\left(a^{*} a-b b^{*}\right)$.

Let $\left\{k_{\ell}\right\}, \ell=1, \ldots, n_{p}$ be a partition of $k, k=\sum_{\ell=1}^{n_{p}} k_{\ell}$. For a given partition, we associate a diagonalization of $k \times k$ matrix $h$;

$$
h=\operatorname{bdiag}\left(\alpha_{1} I_{k_{1}}, \ldots, \alpha_{n_{p}} I_{k_{n_{p}}}\right) \text {, }
$$

with all $\alpha_{\ell}$ are distinct. From $\delta \tau_{1}=\delta \tau_{2}=0, \tau_{1}$ and $\tau_{2}$ becomes $n_{p} \times n_{p}$ block diagonal matrices; $\tau_{1}=\operatorname{bdiag}\left(z_{1}, z_{2}, \ldots, z_{n_{p}}\right), \tau_{2}=\operatorname{bdiag}\left(w_{1}, w_{2}, \ldots, w_{n_{p}}\right)$. For generic choice of $h$, $a$ and $b$ are zero to solve $\delta a=\delta b=0$.

Consider the case $k=(k-l)+l$, the condition $\delta a=0$ is

$$
\left.m\left(\begin{array}{ll}
a_{1,1} & a_{1,2}
\end{array}\right)-\left(\begin{array}{ll}
a_{1,1} & a_{1,2}
\end{array}\right)\left(\begin{array}{cc}
\alpha_{1} & 0 \\
0 & \alpha_{2}
\end{array}\right)=\left(\begin{array}{ll}
m-\alpha_{1}
\end{array}\right) a_{1,1} \quad\left(m-\alpha_{2}\right) a_{1,2}\right)=0
$$

where $a_{1,1}$ and $a_{1,2}$ are $n \times(k-l)$ and $n \times l$ matrices. Non-trivial solutions occur for either $\alpha_{1}=m$ or $\alpha_{2}=m$. We can choose $\alpha_{1}=m$ up to Weyl group of $U(k)$. Then, the condition $\delta a=\delta b=0$ are solved by

$$
a=\left(\begin{array}{ll}
a_{1,1} & 0
\end{array}\right), \quad b=\left(\begin{array}{c}
b_{1,1} \\
0
\end{array}\right)
$$

Now we have

$$
\mu_{\mathbb{C}}=0 \rightarrow\left(\begin{array}{cc}
{\left[z_{1}, w_{1}\right]+b_{1,1} a_{1,1}} & 0 \\
0 & {\left[z_{2}, w_{2}\right]}
\end{array}\right)=0 .
$$

So the fixed point locus is $\hat{\mathcal{M}}_{k-l, n} \times S^{l}\left(\mathbb{C}^{2}\right)$. If we consider the case $k=\sum_{\ell=1}^{n_{p}} k_{\ell}$, we will get one of stratum of $\overline{\mathcal{M}}_{k, n}$ as the fixed point locus.

\section{The Relations with $\mathcal{N}=4$ Super-Yang-Mills Theory}

Now we will explain the original motivation of this paper. To begin with, we rewrite the equations we considered in the previous sections;

$$
\begin{aligned}
\tilde{\mu}_{\mathbb{C}}=\left[t_{1}, t_{2}\right]+\left[t_{3}, t_{4}\right] & =0, \\
\tilde{\mu}_{\mathbb{R}}=\left[t_{1}, t_{1}^{*}\right]+\left[t_{2}, t_{2}^{*}\right]+\left[t_{3}, t_{3}^{*}\right]+\left[t_{4}, t_{4}^{*}\right] & =0,
\end{aligned}
$$


and

$$
\begin{array}{ll}
{\left[t_{5}, t_{1}\right]=0,} & m t_{3}+\left[t_{5}, t_{3}\right]=0, \quad\left[t_{5}, t_{5}^{*}\right]=0, \\
{\left[t_{5}, t_{2}\right]=0, \quad m t_{4}-\left[t_{5}, t_{4}\right]=0,}
\end{array}
$$

where we replaced $t_{0}$, which generate the $U(N)$ symmetry, with $t_{5}$, which generates the $G L(N, \mathbb{C}) .5$ We also supplement the two sets of equations (4.1) and (4.2) with

$$
\left[t_{2}, t_{3}\right]+\left[t_{1}, t_{4}\right]=0, \quad\left[t_{3}, t_{1}\right]+\left[t_{2}, t_{4}\right]=0
$$

These additional equations also do not alter our previous discussions. For example consider a branch of the fixed points given by a partition $N=k+n$ such that

$$
t_{1}=\left(\begin{array}{cc}
z_{1} & 0 \\
0 & z_{2}
\end{array}\right), \quad t_{3}=\left(\begin{array}{cc}
0 & \sigma \\
0 & 0
\end{array}\right), \quad t_{4}=\left(\begin{array}{cc}
0 & 0 \\
\pi & 0
\end{array}\right)
$$

Then (4.3) reduces to

$$
\begin{array}{ll}
z_{2} \sigma=\sigma z_{1}, & z_{1} \sigma=\sigma z_{2}, \\
z_{1} \pi=\pi z_{2}, & z_{2} \pi=\pi z_{1},
\end{array}
$$

For example, the equation for $\sigma$ implies that $\sigma$ should be invariant under the combination of the natural left action of $G L(k, \mathbb{C})$ and the right action $G L(n, \mathbb{C})$. This is entirely consistent with our previous discussions since we reduced everything to the fixed points of the group actions. For this new but equivalent set of equations (4.1), (4.2) and (4.3), the higher dimensional origin of our construction becomes obvious.

\subsection{Dimensional reduction of Vafa-Witten equations}

Now we consider a twisted $\mathcal{N}=4$ super-Yang-Mills theory studied by Vafa and Witten [15]. In the paper [16], we systematically studied the $\mathcal{N}=4$ theory on a Kähler surface and examined how the topological theory changes as one breaks the supersymmetry down to $\mathcal{N}=2$ and $\mathcal{N}=1$. Here I will summarize some relevant results of [15] [16].

On a Kähler surface, the model can be reformulated such that there are 4 topological charges. The resulting theory is an example of the balanced topological field theory [33] with the Dolbeault version of the $\mathcal{G}$-equivariant cohomology. In this model one can introduce $\mathcal{N}=2$ preserving bare mass to the adjoint hyper-multiplet. It turns out that introducing the bare mass is equivalent to combining natural $S^{1}$ actions on the two (twisted) complex scalars in the hyper-multiplet with the $\mathcal{G}$-equivariant cohomology. Consider the

5 Here $t_{5}$ and $t_{5}^{*}$ are They originated from the complex Higgs scalar in the $\mathcal{N}=2$ super-YangMills theory, usually denoted by $\phi$ and $\bar{\phi}$. In the twisted theory it is more natural to regard them as two independent real scalars. Note that $\tilde{\mu}_{\mathbb{R}}$ is the $U(N)$ moment map. 
theory with $U(N)$ gauge group. The equations of the minimum of the bosonic part of the action ), after turning on the bare mass $m$ (equivalently, after breaking $\mathcal{N}=4$ down to $\mathcal{N}=2$ supersymmetry) are given by

$$
\begin{aligned}
F_{A_{0}}^{0,2}+\left[t_{3}, t_{4}\right] & =0, \quad \partial_{A} t_{5}=\bar{\partial}_{A} t_{5}=0, \quad \bar{\partial}_{A}^{*} t_{3}+\bar{\partial}_{A} t_{4}=0 \\
F_{A_{0}} \wedge \omega+\left[t_{3}, t_{3}^{*}\right]+\left[t_{4}, t_{4}^{*}\right] \omega^{2} & =0,
\end{aligned}
$$

together with

$$
\begin{aligned}
& m t_{3}+\left[t_{5}, t_{3}\right]=0, \\
& m t_{4}-\left[t_{5}, t_{4}\right]=0 \text {, } \\
& {\left[t_{5}, t_{5}^{*}\right]=0 .}
\end{aligned}
$$

Note that the above two sets of equations (4.6) and (4.7) are fixed points of the global supersymmetry of the twisted $\mathcal{N}=4$ super-Yang-Mills after turning on the bare mass.

Here we write $F_{A_{0}}$ for the trace free part, i.e. $F_{A}=F_{A_{0}}+\frac{1}{N} \operatorname{tr} F_{A} \otimes I_{N}$. We also have $\operatorname{tr} F_{A}^{+}=\omega^{+}$for a self-dual two form $2 \pi i \omega^{+}=c_{1}(E)$. One may identify $t_{5}$ with a scalar in the $\mathcal{N}=2$ vector multiplets, $t_{3}$ and $t_{4}$ as the two complex scalars in the $\mathcal{N}=2$ hyper-multiplet. After twisting $t_{3}$ becomes the holomorphic two form. Note that every scalar fields carry the adjoint representation of $U(N)$. The equation (4.6) is nothing but the Vafa-Witten equation in the complex coordinates. The equations (4.6) are invariant under the $S^{1}$ actions $\left(t_{3}, t_{4}\right) \rightarrow\left(e^{i m \varphi} t_{4}, e^{-i m \varphi} t_{4}\right)$. This originates from the unbroken global symmetry of the twisted $\mathcal{N}=4$ theory [15] and identical to the $S_{m}^{1}$ action studied in Sect. 3.1.

Together with 4 components of the connection, we have 10 matrix-valued forms. If we do dimensional reduction of the above equations down to 0-dimension, we precisely get our equations (4.1) (4.2) (4.3) for extended monads. In other words, the dimensional reduction of $\mathcal{N}=2$ preserving $\mathcal{N}=4$ super-Yang-Mills theory reduces the infinite dimensional $\mathcal{G} \times S^{1}$-equivariant cohomology of the space of connections with additional matter fields to the finite dimensional $U(N) \times S^{1}$-equivariant cohomology of the extended monads. And the both equation describe Yang-Mills instantons.6 This is an amazing property stems from reciprocity.

\subsubsection{Perturbation to $\mathcal{N}=1$ theory and the resolution of the singularities}

In the paper of Vafa and Witten [15], a perturbation of $\mathcal{N}=4$ theory to $\mathcal{N}=1$ theory was crucial. If we break the supersymmetry further down to $\mathcal{N}=1$, the classical vacua are classified by the complex conjugacy classes of homomorphisms of the $S U(2)$ Lie algebra to

6 Note that the Vafa-Witten equation (4.6) on $\mathbb{R}^{4}$ enjoys certain vanshing theorem such that it reduces to the $\mathrm{ASD}$ equation [15]. 
that of $U(N)$ [15]. In that case, the first column of (4.6) is changed in a very interesting way;

$$
\begin{array}{r}
F_{A_{0}}^{0,2}+\left[t_{3}, t_{4}\right]+t_{5}^{0} \omega^{0,2}=0, \\
\operatorname{tr} F_{A}^{0,2}+\operatorname{tr} t_{5} \omega^{0,2}=\left(\omega^{+}\right)^{0,2},
\end{array}
$$

where $\omega^{0,2}$ is the holomorphic two-form which corresponds to the $\mathcal{N}=1$ preserving bare mass and $t_{5}^{0}$ is the trace-free part of $t_{5}$. In the paper [16], we use the above to recover Witten's judicious perturbation of the Abelian Seiberg-Witten equation [34]. In other words, the perturbation considered by Vafa and Witten [15] becomes identical to Witten's perturbation of abelian Seiberg-Witten equation at a fixed point of the $S^{1}$ action. Such perturbation leads to a factorization of Seiberg-Witten class which is crucial in finding all the basic classes [34]. Mathematically such perturbation ensure the moduli space of abelian Seiberg-Witten monopoles behaves well.

Now we dimensionally reduce the perturbed equation down to zero. Then, the equations in (4.1) becomes

$$
\begin{aligned}
& \tilde{\mu}_{\mathbb{C}}=\left[t_{1}, t_{2}\right]+\left[t_{3}, t_{4}\right]=-t_{5}^{0}, \\
& \tilde{\mu}_{\mathbb{R}}=\left[t_{1}, t_{1}^{*}\right]+\left[t_{2}, t_{2}^{*}\right]+\left[t_{3}, t_{3}^{*}\right]+\left[t_{4}, t_{4}^{*}\right]=0,
\end{aligned}
$$

Note that the real equation in (4.1) and the remaining equations (4.2)(4.3) remain unchanged.

Now we consider solutions of (4.9) together with equations (4.2) (4.3). This is equivalent to considering the fixed points of $S_{m}^{1}$ action discussed in Sect. 3.2. For simplicity, we consider the case of example (2), we have (the modification for (3.25));

$$
\left\{\begin{array} { l } 
{ [ z _ { 1 } , w _ { 1 } ] + \sigma \pi = - \alpha I _ { k } , } \\
{ [ z _ { 1 } , z _ { 1 } ^ { * } ] + [ w _ { 1 } , w _ { 1 } ^ { * } ] + \sigma \sigma ^ { * } - \pi ^ { * } \pi = 0 , }
\end{array} \quad \left\{\begin{array}{l}
{\left[z_{2}, w_{2}\right]-\pi \sigma=\frac{k \alpha}{n} I_{n},} \\
{\left[z_{2}, z_{2}^{*}\right]+\left[w_{2}, w_{2}^{*}\right]+\pi \pi^{*}-\sigma^{*} \sigma=0,}
\end{array}\right.\right.
$$

with $(1+k / n) \alpha=m$. For example, the first equation says the fixed point locus is

$$
\overline{\mathcal{M}}_{n, k}^{\varepsilon_{\mathbb{C}} \alpha}=\mu_{\mathbb{R}}^{-1}(0) \cap \mu_{\mathbb{C}}^{-1}\left(-\alpha I_{k}\right) / U(k) \equiv \mu_{\mathbb{C}}^{-1}\left(-\alpha I_{k}\right) / / G L(k, \mathbb{C}) .
$$

This fits nicely with the variation of GIT quotients such that the above can be viewed as a resolution of the singularities [35] of the completed moduli space of framed instantons:

$$
\overline{\mathcal{M}}_{n, k}=\mu_{\mathbb{R}}^{-1}(0) \cap \mu_{\mathbb{C}}^{-1}(0) / U(k)=\bigcup_{\ell=0}^{k}\left(\hat{\mathcal{M}}_{k-\ell, N-k} \times S^{\ell}\left(\mathbb{C}^{2}\right)\right) .
$$

It is amusing to see that the same prescription in $\mathcal{N}=4$ SYM theory leads both the moduli space of Seiberg-Witten monopoles and the completed moduli space of framed instantons. 


\subsection{2. $S^{1}$ Actions invisible in the Vafa-Witten equation}

We saw that the extended monads considered in Sect. 3.1 are directly related with the $\mathcal{N}=4$ super-Yang-Mills theory in 4-dimensions. The $S_{m}^{1}$ action consider in Sect. 3.2 was precisely motivated by the $\mathcal{N}=4 \mathrm{SYM}$ theory, which has the same global symmetry and the bare mass is related to the generator of the same $S_{m}^{1}$ action. A crucial difference from the Vafa-Witten equation (4.6) and the equations for the extended monads (4.1) with (4.9) is that the later has another $S^{1} \times S^{1}$ action;

$$
\left(t_{1}, t_{2}, t_{3}, t_{4}\right) \rightarrow\left(e^{i \varrho^{\prime}} t_{1}, e^{i \varrho^{\prime \prime}} t_{2}, e^{i \varrho^{\prime}} t_{3}, e^{i \varrho^{\prime \prime}} t_{4}\right) .
$$

This action preserve $\tilde{\mu}_{\mathbb{R}}$ while the complex momentum map gets the phase factor $\tilde{\mu}_{\mathbb{C}} \rightarrow$ $e^{i\left(\varrho^{\prime}+\varrho^{\prime \prime}\right)} \tilde{\mu}_{\mathbb{C}}$.

Now the space of the extended monads has $U(N) \times S_{m}^{1} \times S_{\varrho^{\prime}}^{1} \times S_{\varrho^{\prime \prime}}^{1}$, which preserve the Kähler potential $\mathfrak{k}$ given by (3.11). This leads to the following modification of the basic supersymmetry algebra (3.14)

$$
\begin{array}{ll}
\mathbf{s} t_{j}=\psi_{j}, & \mathbf{s} \psi_{j}=0, \\
\overline{\mathbf{s}} t_{j}=0, & \overline{\mathbf{s}} \psi_{j}=-i\left[t_{0}, t_{j}\right]+i \varrho^{\prime} t_{j}\left(\delta_{1 j}+\delta_{3 j}\right)+i \varrho^{\prime \prime} t_{j}\left(\delta_{2 j}+\delta_{4 j}\right)-i m t_{j}\left(\delta_{3 j}-\delta_{4 j}\right), \\
\mathbf{s} t_{j}^{*}=0, & \mathbf{s} \psi_{j}^{*}=-i\left[t_{0}, t_{j}^{*}\right]-i \varrho^{\prime} t_{j}^{*}\left(\delta_{1 j}+\delta_{3 j}\right)-i \varrho^{\prime \prime} t_{j}^{*}\left(\delta_{2 j}+\delta_{4 j}\right)+i m t_{j}^{*}\left(\delta_{3 j}-\delta_{4 j}\right), \\
\overline{\mathbf{s}} t_{j}^{*}=\psi_{j}^{*}, & \overline{\mathbf{s}} \psi_{j}^{*}=0,
\end{array}
$$

where $j=1,2,3,4$. Then one find the equivariant Kähler form as

$-\frac{i}{2}(\mathbf{s} \overline{\mathbf{s}}-\overline{\mathbf{s}} \mathbf{s}) \mathfrak{k}=-\frac{1}{2} \operatorname{tr}\left(t_{0} \tilde{\mu}_{\mathbb{R}}+m\left(t_{3} t_{3}^{*}-t_{4} t_{4}^{*}\right)+\varrho^{\prime}\left(t_{1} t_{1}^{*}+t_{3} t_{3}^{*}\right)+\varrho^{\prime \prime}\left(t_{2} t_{2}^{*}+t_{4} t_{4}^{*}\right)-i \sum_{j} \psi_{j} \psi_{j}^{*}\right)$.

One can identify the first 4 terms with the momentum maps of $U(N), S_{m}^{1}, S_{\varrho^{\prime}}^{1}$ and $S_{\varrho^{\prime \prime}}^{1}$ actions, respectively. Note that the Hamiltonian,

$$
-\frac{1}{2} \operatorname{tr}\left(\varrho^{\prime}\left(t_{1} t_{1}^{*}+t_{3} t_{3}^{*}\right)+\varrho^{\prime \prime}\left(t_{2} t_{2}^{*}+t_{4} t_{4}^{*}\right)\right)
$$

of $S_{\varrho^{\prime}}^{1} \times S_{\varrho^{\prime \prime}}^{1}$ action is proportional to the Kähler potential $\mathfrak{k}$ for $\varrho^{\prime}=\varrho^{\prime \prime}$. We can use the above as a Morse function to study homology. The critical points of such a Morse function are identical to the fixed point of $\mathbf{s}$ and $\overline{\mathbf{s}}$ in (4.14). Physically, including those $S^{1}$ actions corresponds to giving masses to all the fields. This is not possible in general for its field theoretical cousins without breaking the gauge symmetry. Now we can conclude that any homological problem concerning our extended moduli space can be localized to the problem on the fixed point locus of above. This additional symmetry should greatly simplify any approach based on the space of monads.

7 The choice of the $S_{\varrho^{\prime}}^{1} \times S_{\varrho^{\prime \prime}}^{1}$ action has made to preserve the equations (4.3), which is the dimensional reduction of the Dirac equations for the two complex fields in the hyper-multiplet. The later can be also viewed as a momentum map. 


\subsection{A Matrix Model}

Physically, we can use (4.15) as an action functional. Then we have a supersymmetric matrix integral analog to the path integral integral. Provided that we restrict to configurations satisfying $\tilde{\mu}_{\mathbb{C}}=0$, which can easily be implemented supersymmetrically, we have

$$
\begin{aligned}
\int_{\overline{\mathfrak{M}}_{N}^{1,1}} & d^{N^{2}} t_{0}\left(\prod_{i=1}^{4} d^{N^{2}} t_{i} d^{N^{2}} t_{i}^{*} d^{N^{2}} \psi_{i} d^{N^{2}} \psi_{i}^{*}\right) \\
& \times e^{-\frac{1}{2} \operatorname{tr}\left(t_{0} \tilde{\mu}_{\mathbb{R}}+m\left(t_{3} t_{3}^{*}-t_{4} t_{4}^{*}\right)+\varrho^{\prime}\left(t_{1} t_{1}^{*}+t_{3} t_{3}^{*}\right)+\varrho^{\prime \prime}\left(t_{2} t_{2}^{*}+t_{4} t_{4}^{*}\right)-i \sum_{j} \psi_{j} \psi_{j}^{*}\right)}
\end{aligned}
$$

Integration over $t_{0}$ leads to an algebraic equation of motion $\mu_{\mathbb{R}}=0$. The integration over $\psi_{i}$ and $\psi_{i}^{*}$ endows $\overline{\mathfrak{A}}_{N}^{1,1}$ with the symplectic measure. Then the remaining integration is precisely the Duistermatt-Heckmann $(\mathrm{DH})$ integration formula for the $S_{m}^{1} \times S_{\varrho^{\prime}}^{1} \times S_{\varrho^{\prime \prime}}^{1}$ actions [36]. So the integration is localized to the fixed points and can be evaluated exactly. Note as one of fixed point locus of $S_{m}^{1}$ action we get the completed moduli space of certain framed instantons.

For the particular integral (4.17) we have a natural prescription for resolving the singularities. Note that $\mathbf{s} t_{0}=\overline{\mathbf{s}} t_{0}=0$. We can form an obvious observable, for example, $-\frac{1}{4} \operatorname{tr} t_{0}^{2}$. Now we define a new matrix integral

$$
\begin{aligned}
\int_{\overline{\mathfrak{A}}_{N}^{1,1}} & d^{N^{2}} t_{0}\left(\prod_{i=1}^{4} d^{N^{2}} t_{i} d^{N^{2}} t_{i}^{*} d^{N^{2}} \psi_{i} d^{N^{2}} \psi_{i}^{*}\right) \\
& \times e^{-\frac{1}{2} \operatorname{tr}\left(t_{0} \tilde{\mu}_{\mathbb{R}}+m\left(t_{3} t_{3}^{*}-t_{4} t_{4}^{*}\right)+\varrho^{\prime}\left(t_{1} t_{1}^{*}+t_{3} t_{3}^{*}\right)+\varrho^{\prime \prime}\left(t_{2} t_{2}^{*}+t_{4} t_{4}^{*}\right)-i \sum_{j} \psi_{j} \psi_{j}^{*}\right)-\frac{\varepsilon}{4} \operatorname{tr} t_{0}^{2}} .
\end{aligned}
$$

The algebraic equations of of above action functional are given by

$$
\tilde{\mu}_{\mathbb{R}}=-\varepsilon t_{0}, \quad \overline{\mathbf{s}} \psi_{j}=\mathbf{s} \psi_{j}^{*}=0 .
$$

Physically introducing an $\varepsilon$ dependent term regularize the path integral. The norm squared of momentum map $-\operatorname{tr}\left(\tilde{\mu}_{\mathbb{R}}^{2}\right)$ has no higher critical points. So introducing $\frac{\varepsilon}{4} \operatorname{tr}\left(t_{0}^{2}\right)$ does not introduce any new fixed points. Instead it resolve the singularities of the fixed point locus of $S_{m}$ action. Thus we have another resolution of singularity by the perturbation;

$$
\tilde{\mu}_{\mathbb{C}}=0, \quad \tilde{\mu}_{\mathbb{R}}=-\varepsilon t_{0} .
$$

Now Witten's fixed point theorem ensures that the matrix integral (4.18) is localized on the solutions of (4.20) together with the fixed points of supersymmetry $\mathbf{s}$ and $\overline{\mathbf{s}}$ in (4.14). 
Clearly, the fixed points are identical to the fixed point of the torus action (4.13). If we consider a fixed point of the $S_{m}^{1}$ action only as example (2), we have

$$
\left\{\begin{array} { l } 
{ [ z _ { 1 } , w _ { 1 } ] + \sigma \pi = 0 , } \\
{ [ z _ { 1 } , z _ { 1 } ^ { * } ] + [ w _ { 1 } , w _ { 1 } ^ { * } ] + \sigma \sigma ^ { * } - \pi ^ { * } \pi = - \varepsilon \alpha _ { \mathbb { R } } I _ { k } , }
\end{array} \left\{\begin{array}{l}
{\left[z_{2}, w_{2}\right]-\pi \sigma=0,} \\
{\left[z_{2}, z_{2}^{*}\right]+\left[w_{2}, w_{2}^{*}\right]+\pi \pi^{*}-\sigma^{*} \sigma=\varepsilon \frac{k \alpha_{\mathbb{R}}}{n} I_{n},}
\end{array}\right.\right.
$$

with $(1+k / n) \alpha=m$. This is the real perturbation related with the complex perturbation (4.10). The solution space of the first set of equation in (4.21) is

$$
\overline{\mathcal{M}}_{n, k}^{\varepsilon_{\mathbb{R}} \alpha}=\mu_{\mathbb{R}}^{-1}\left(-\varepsilon_{\mathbb{R}} \alpha I_{k}\right) \cap \mu_{\mathbb{C}}^{-1}(0) / U(k) .
$$

In this case the resolution of the singularities occurs in the framework of the variation of the symplectic quotients [37].

We note that the above integral is of the form of the $U(N)$ equivariant integration of Witten [38]. Jefferey and Kirwan proved a version of Witten's non-Abelian localization such that the integral is localized to the fixed points of the action of the maximal torus of $U(N)$ [39. Together with the DH type localization, the integral (4.18) is localized on the fixed points of $T^{N} \times S_{m}^{1} \times S_{\varrho^{\prime}}^{1} \times S_{\varrho^{\prime \prime}}^{1}$ actions. In principle, the integral (4.18) should be exactly computable.

One can also do a Morse theory to evaluate the Poincaré polynomial of $\overline{\mathfrak{M}}_{N}^{t_{0}}$. Physically, this amounts to study the balanced topological theory [33]. The contribution of a particular fixed point locus of the $S_{m}^{1}$ action is identical to the partition function of $\mathcal{N}=4$ super-Yang-Mills theory in four-dimensions with some gauge group and instanton number. Surprisingly, such a theory is closely related to the dimensional reduction of $\mathcal{N}=4$ superYang-Mills theory all the way down to zero-dimension. The large $N$ limit of such balanced theory should be also compared with the matrix model approaches [40] for $M$ theory [41].

The details will appear in a forthcoming paper [42].

\section{Some speculative remarks}

It will be very interesting to examine the $N \rightarrow \infty$ limit of (4.18). Then, the fixed point locus of $S_{m}^{1}$ action contains an arbitrary produc of resolved and completed moduli spaces of $S U(n)$ instantons with all ranks $n$ and all instanton numbers. This should be compared with the field theoretical approach where one fixes a gauge group and sums over the contributions of all instanton numbers. In the matrix integral (4.18), contribution from a particular fixed can be identified with the Donaldson invariants or topological correlation function of $\mathcal{N}=2$ super-Yang-Mills theory in four-dimensions [43] 44]. Our matrix integral puts the rank of gauge group and the instanton number on equal footing and considers all possible moduli spaces simultaneously. Due to the original suggestion of t' Hooft, we expect 
the physics becomes simplified or has new noble properties by taking $n \rightarrow \infty$ limit [45]. However, the reciprocity, which interchange $n$ with $k$ for the instanton contributions, seems to suggest us to reexamine the physics of $n \rightarrow \infty$ limit. The viewpoint we developed here seems to suggest that we better consider all values of rank $n$ and the instanton number $k$ simultaneously. Presumably, the genuine stringy property of QCD becomes more manifest in the $N \rightarrow \infty$ limit in the new setting than in the usual large $n$ limit.

Consider Yang-Mills instantons on an ALE space (an ALE gravitational instanton). Let $\mathfrak{N}(\mathbf{u}, \mathbf{w})$ denote a completed moduli space of framed instantons, classified by the Mukai vectors $\mathbf{u}$ and $\mathbf{v}$, after resolving the singularities. Nakajima constructed irreducible representations of affine Lie algebra using certain homology class of $\mathfrak{N}$ [32] 46]. An important thing in his construction is a Lagrangian subvariety $\mathfrak{N}(\mathbf{u}, \mathbf{w})$ in the product of two-moduli spaces, $\mathfrak{N}\left(\mathbf{u}+\mathbf{C e}^{i}, \mathbf{w}\right) \times \mathfrak{N}(\mathbf{u}, \mathbf{w})$. This Lagrangian subvariety is closely related with another Lagrangian subvarieties in the moduli spaces $\mathfrak{N}\left(\mathbf{u}+\mathbf{C e}^{i}, \mathbf{w}\right)$ and $\mathfrak{N}\left(\mathbf{u}^{i}, \mathbf{w}\right)$. The later subvarieties are defined by the compact sets of gradient flows between the critical points of Hamiltonian analogous to (4.16). Note that we get, as a fixed point of $S_{m}^{1}$ action, various product spaces of completed moduli space of framed instantons on $\mathbb{R}^{4}$ with resolved singularities. So we automatically get the natural setting for Nakajima's story. One may start from examining the gradient flow between the critical points of (4.16).

We also like to point out that the matrix integral (4.18) with the invariant polynomial $V\left(t_{0}\right)=\sum_{j=2}^{N} \frac{\varepsilon_{j}}{2 j !} \operatorname{tr}\left(t_{0}^{j}\right)$ has a great similarity with the random matrix model (See, for example, a collection of the original papers [47]). It would be certainly possible that the matrix integral enjoys a recursion relation for different values of $N$.

\subsubsection{The original ADHM case}

Before leaving this section, we will consider the case of the original ADHM construction. This is relevant since such a case appears as a particular fixed point of our model.

The $S_{\varrho^{\prime}}^{1} \times S_{\varrho^{\prime \prime}}^{1}$ action (4.13) descends to the fixed point locus of the $S_{m}^{1}$ action as considered in Sect. 3.2. On the moduli space $\overline{\mathcal{M}}_{n, k}^{\alpha_{\mathbb{R}}}$ described $\left(\tau_{1}, \tau_{1}, a, b\right)$, for example in (4.10), the action becomes

$$
\left(\tau_{1}, \tau_{2}, a, b\right) \rightarrow\left(e^{i \varrho^{\prime}} \tau_{1}, e^{i \varrho^{\prime \prime}} \tau_{2}, e^{i \varrho^{\prime}} a, e^{i \varrho^{\prime \prime}} b\right)
$$

This action preserve the induced Käher potential $\tilde{\kappa}$ on $\overline{\mathcal{M}}_{n, k}^{\alpha_{\mathbb{R}}}$;

$$
\tilde{\kappa}=-\frac{1}{2} \operatorname{tr}\left(\tau_{1} \tau_{1}^{*}+\tau_{2} \tau_{2}^{*}+a^{*} a+b b^{*}\right)
$$


On the fixed point locus $\overline{\mathcal{M}}_{n, k}^{\alpha_{\mathbb{R}}}$, (4.14) reduces to

$$
\begin{array}{ll}
\mathbf{s} \tau_{j}=\chi_{j}, & \overline{\mathbf{s}} \chi_{j}=-i\left[\tau_{0}, \tau_{j}\right]+i \varrho^{\prime} \tau_{j} \delta_{1 j}+i \varrho^{\prime \prime} \tau_{j} \delta_{2 j}, \\
\overline{\mathbf{s}} \tau_{j}^{*}=\chi_{j}^{*}, & \mathbf{s} \chi_{j}^{*}=-i\left[\tau_{0}, \tau_{j}^{*}\right]-i \varrho^{\prime} \tau_{j}^{*} \delta_{1 j}-i \varrho^{\prime \prime} \tau_{j}^{*} \delta_{2 j},
\end{array}
$$

where $j=1,2$ and

$$
\begin{array}{ll}
\mathbf{s} a=\mathfrak{a}, & \overline{\mathbf{s}} \mathfrak{a}=+i a \tau_{0}+i \varrho^{\prime} a, \\
\overline{\mathbf{s}} a^{*}=\mathfrak{a}^{*}, & \mathbf{s} \mathfrak{a}^{*}=-i \tau_{0} a^{*}-i \varrho^{\prime} a^{*}, \\
\mathbf{s} b=\mathfrak{b}, & \overline{\mathbf{s}} \mathfrak{b}=-i \tau_{0} b+i \varrho^{\prime \prime} b, \\
\overline{\mathbf{s}} b^{*}=\mathfrak{b}^{*}, & \mathbf{s b}^{*}=+i b^{*} \tau_{0}-i \varrho^{\prime \prime} b^{*},
\end{array}
$$

where $\tau_{0}$ denotes the generator of $U(k)$ action $\left(\tau_{1}, \tau_{2}, a, b\right) \rightarrow\left(g \tau_{1} g^{-1}, g \tau_{2} g^{-1}, a g^{-1}, g b\right)$ where $g \in U(k)$. The equivariant Kähler form is given by

$$
\begin{aligned}
-\frac{i}{2}(\mathbf{s} \overline{\mathbf{s}}-\overline{\mathbf{s}} \mathbf{s}) \tilde{\kappa}= & -\frac{1}{2} \operatorname{tr}\left(\tau_{0} \mu_{\mathbb{R}}\right)+-\frac{1}{2} \operatorname{tr}\left(\varrho^{\prime} \tau_{1} \tau_{1}^{*}+\varrho^{\prime \prime} \tau_{2} \tau_{2}^{*}+\varrho^{\prime} a^{*} a+\varrho^{\prime \prime} b b^{*}\right) \\
& +i \operatorname{tr}\left(\sum_{j} \chi_{j} \chi_{j}^{*}+\mathfrak{a}^{*} \mathfrak{a}+\mathfrak{b} \mathfrak{b}^{*}\right)
\end{aligned}
$$

Provided that the restriction to $\mu_{\mathbb{C}}=0$ is understood, we can use the equivariant Kähler form (4.27) as the action functional. We define

$$
\begin{aligned}
S\left(\varepsilon, \varrho^{\prime}, \varrho^{\prime \prime}\right)= & \frac{1}{2} \operatorname{tr}\left(\tau_{0} \mu_{\mathbb{R}}\right)+\frac{1}{2} \operatorname{tr}\left(\varrho^{\prime} \tau_{1} \tau_{1}^{*}+\varrho^{\prime \prime} \tau_{2} \tau_{2}^{*}+\varrho^{\prime} a^{*} a+\varrho^{\prime \prime} b b^{*}\right) \\
& -i \operatorname{tr}\left(\sum_{j} \chi_{j} \chi_{j}^{*}+\mathfrak{a}^{*} \mathfrak{a}+\mathfrak{b} \mathfrak{b}^{*}\right)-\frac{\varepsilon}{4} \operatorname{tr} \tau_{0}^{2} .
\end{aligned}
$$

Here, $\tau_{0}$ is the generator of $U(k)$ action and the role of the $\varepsilon$ dependent term is the resolution of the singularities in $\hat{\mathcal{M}}_{k, n}$; the $\tau_{0}$ equation of motion is

$$
\mu_{\mathbb{R}}=\left[\tau_{1}, \tau_{1}^{*}\right]+\left[\tau_{2}, \tau_{2}^{*}\right]+b b^{*}-a^{*} a=\varepsilon \tau_{0} .
$$

This gives us a constraint

$$
\operatorname{tr}\left(b b^{*}-a a^{*}\right)=\varepsilon \operatorname{tr}\left(t_{0}\right) .
$$

The term $i \operatorname{tr}\left(\sum_{j} \chi_{j} \chi_{j}^{*}-\mathfrak{a}^{*} \mathfrak{a}-\mathfrak{b} \mathfrak{b}^{*}\right)$ is the Kähler form of $\hat{\mathcal{M}}_{k, n}$. The role of the $\varepsilon$ dependent term is the resolution of the singularities in $\hat{\mathcal{M}}_{k, n}$. The momentum map of $S_{\varrho^{\prime}}^{1} \times S_{\varrho^{\prime \prime}}^{1}$ is given by

$$
-\frac{1}{2} \operatorname{tr}\left(\varrho^{\prime} \tau_{1} \tau_{1}^{*}+\varrho^{\prime \prime} \tau_{2} \tau_{2}^{*}+\varrho^{\prime} a^{*} a+\varrho^{\prime \prime} b b^{*}\right)
$$


which critical points are identical to the fixed points of supersymmetry in (4.25) (4.26). The matrix integral with the action functional (4.28) is localized on, after applying the non-abelian localization, the fixed point of $T^{k} \times S_{\varrho^{\prime}}^{1} \times S_{\varrho^{\prime \prime}}^{1}$.

If we set $\varrho^{\prime}=\varrho^{\prime \prime}=0$, the above action functional is closely related to the action functional of holomorphic Yang-Mills theory 48];

$$
I(\varepsilon)=-\frac{i}{4 \pi^{2}} \int \operatorname{tr}\left(i \varphi F_{A}\right) \wedge \omega-\frac{1}{4 \pi^{2}} \int \operatorname{tr}(\psi \wedge \bar{\psi}) \wedge \omega-\frac{\varepsilon}{8 \pi^{2}} \int \operatorname{tr}\left(\varphi^{2}\right),
$$

where $\omega$ denotes the Kähler form. Here $\phi$ is the generator of infinite dimensional gauge symmetry $\mathcal{G}$. The second term $\frac{1}{4 \pi^{2}} \int \operatorname{tr}(\psi \wedge \bar{\psi}) \wedge \omega$ is identical to the $L^{2}$ Kähler form of $\mathcal{M}_{n, k}$. Due to the isometry mentioned earlier the two theories are equivalent. In the field theoretical approach, however, we do not know how to complete the metric in the moduli space. The approach based on the ADHM construction is more simpler and well-defined. Furthermore we can also utilize the $S_{\varrho^{\prime}}^{1} \times S_{\varrho^{\prime \prime}}^{1}$ actions, which are invisible in the field theory cousin.

We can also do Morse theory using the Hamiltonian (4.31). This amounts to considering the balanced topological theory based on the ADHM data. The resulting theory is equivalent to $\mathcal{N}=4$ super-Yang-Mills theory which suffer the similar problems like holomorphic Yang-Mills theory. The details will appear in [42].

In the papers [46], Nakajima studied the resolution of singularities in the completed moduli space of framed instantons, say $\overline{\mathcal{M}}_{n, k}$, on $\mathbb{C}^{2}$. He rigorously showed that the perturbation leads to a resolution of the singularities of (4.12). He also studied the $S_{\varrho^{\prime}}^{1} \times S_{\varrho^{\prime \prime}}^{1}$ actions (4.23) and determined the fixed points and the Morse index. The momentum map (4.31) turns a perfect Morse functional. Moreover, the critical point set consists of finite points [49]. This amounts to providing an algorithm to compute the Poincaré polynomial of $\overline{\mathcal{M}}_{n, k}^{\alpha_{\mathbb{R}} T_{k}}$. See [50] for a model computation for the rank $n=1$ case (the rank 1 torsionfree sheaves). Here we recovered Nakajima's approach in terms of natural field theoretical method.

\section{D-instantons, Reciprocity and D-branes}

We have developed enough material to come to the main theme of this paper, Dinstantons.

To begin with, We recall some properties of the D-instanton following Witten [14]. The Type IIB string can have (-1)-branes or D-instantons. The low energy effective theory of $N$ D-instantons y are given by the dimensional reduction of $\mathcal{N}=1 d=10$ superYang-Mills theory with $U(N)$ gauge group all the way down to zero-dimension. For the 
supersymmetric ground state of the D-instantons are determined by ten $U(N)$ matrices $X^{i}$ with

$$
\left[X_{i}, X_{j}\right]=0, \quad i, j=1, \ldots, 10
$$

This is the absolute minimum of the bosonic part of the action

$$
I=\frac{T^{2}}{2} \sum_{i<j} \operatorname{tr}\left[X^{i}, X^{j}\right]^{2}+\ldots
$$

Now for classical states of the unbroken supersymmetry given by (5.1), the matrices can be diagonalized simultaneously. The eigenvalues of $X_{i}$ can be interpreted as the positions of $N$ unordered (identical) points. Expanding around the classical vacua, one interprets the off-diagonal components as representing strings joining D-instantons. The classical moduli space of D-instantons is $S^{N}\left(\mathbb{R}^{10}\right)$. The moduli space is singular when more than two D-instantons coincide. Such a singularity corresponds to certain gauge symmetry enhancement. As the $n$ of the D-instantons coincide the gauge symmetry is enhanced to $U(n)$.

As pointed out by Witten [14], the above descriptions of D-instanton has an intriguing similarity with the ADHM description of the Yang-Mills instantons, although the governing equations are different. The situation is just like the ADHM description of the point-like instantons, which correspond to completely degenerated instantons. In this section we will discuss relations between D-instantons and Yang-Mills instantons using the dimensional reduction of twisted $\mathcal{N}=4$ super-Yang-Mills theory with gauge group $U(N)$ in 4-dimension down to 0-dimension.

\subsection{D-instantons and Yang-Mills instantons}

We will consider supersymmetric ground states of D-instantons described by the equations (4.1), (4.2) and (4.3).8 We are interested in the case that half of the supersymmetry breaks down after turning on the bare mass $m$ in (4.2). We already studied the corresponding supersymmetric ground states in the previous sections. The classical vacua are classified by the eigenvalues of the diagonalized matrix $t_{5}$. Assume, for simplicity, the case of a partition $N=k+n$ such that $t_{5}=\operatorname{diag}\left(\alpha I_{k}, \beta I_{n}\right)$, the example (2) of Sect. 3.3.1. Then the diagonal elements of of $t_{3}$ and $t_{4}$ are identically zero. This corresponds to the case that $k$ D-instantons are confined in a 2-complex dimensional hyperplane defined by $t_{3}=t_{4}=0, t_{5}=\alpha$ and $n$ D-instantons are confined in another hyperplane defined by $t_{3}=t_{4}=0, t_{5}=\beta$.

8 We will use complex notation such that $t_{j}=X_{2 j-1}+i X_{2 j}$ for $j=1, \ldots, 5$. 
If $|\alpha-\beta| \neq m$, the off-diagonal elements of $t_{3}$ and $t_{4}$ also vanish identically. The fixed point equation we get is

$$
\begin{array}{ll}
{\left[z_{1}, w_{1}\right]=0,} & {\left[z_{2}, w_{2}\right]=0,} \\
{\left[z_{1}, z_{1}^{*}\right]+\left[w_{1}, w_{1}^{*}\right]=0,} & {\left[z_{2}, z_{2}^{*}\right]+\left[w_{2}, w_{2}^{*}\right]=0,}
\end{array}
$$

where $z_{1}, w_{1}$ are $k \times k$ and $z_{2}, w_{2}$ are $n \times n$ matrices. In terms of the ADHM description of Yang-Mills instantons we end up with $k$ and $n$ point-like instantons in the first and the second hyperplanes, repectively. Their positions in the two hyperplanes are given by the eigenvalues of $\left(z_{1}, w_{1}\right)$ and $\left(z_{2}, w_{2}\right)$ respectively.

Now let $|\alpha-\beta|=m$, then the off-diagonal parts of $t_{3}$ and $t_{4}$ appears as given by (3.24). We have

$$
\left\{\begin{array} { l } 
{ [ z _ { 1 } , w _ { 1 } ] + \sigma \pi = 0 , } \\
{ [ z _ { 1 } , z _ { 1 } ^ { * } ] + [ w _ { 1 } , w _ { 1 } ^ { * } ] + \sigma \sigma ^ { * } - \pi ^ { * } \pi = 0 , }
\end{array} \quad \left\{\begin{array}{l}
{\left[z_{2}, w_{2}\right]-\pi \sigma=0,} \\
{\left[z_{2}, z_{2}^{*}\right]+\left[w_{2}, w_{2}^{*}\right]+\pi \pi^{*}-\sigma^{*} \sigma=0,}
\end{array}\right.\right.
$$

where $\sigma$ is $k \times n$ and $\pi$ is $n \times k$ matrices. We also have

$$
\operatorname{tr} \sigma \pi=\operatorname{tr} \pi \sigma=0, \quad \operatorname{tr} \sigma \sigma^{*}=\operatorname{tr} \pi^{*} \pi, \quad \operatorname{tr} \sigma^{*} \sigma=\operatorname{tr} \pi \pi^{*} .
$$

The first set of equations in (5.4) corresponds to the $k S U(n)$ instantons in the first hyperplane and the second one does the $n S U(k)$ instantons in the second hyperplane. Note that the role of $\sigma$ and $\pi$ are exchanged in the two sets of the equations in (5.4).

Now we consider the role of the off-diagonal parts $\sigma$ and $\pi$ of $t_{3}$ and $t_{4}$, respectively. Here we should distinguish the non-degenerate instantons from the degenerate one. First we consider the non-degenerate case so that we do not have any point-like instantons. Note the non-degeneracy condition means that every matrix element of the $n \times k$ matrices $\pi$ and $\sigma^{*}$ is non-zero. The matrices $\pi$ and $\sigma^{*}$ can naturally be interpreted as representing oriented strings starting from the $k$ D-instantons in first hyperplane and ending on the $n$ D-instantons in the second hyperplane. The $k \times n$ matrices $\sigma$ and $\pi^{*}$ correspond to strings starting from the D-instantons $n$ in the second hyperplane and ending on the $k$ D-instantons in the first hyperplane. Note also that the $\operatorname{tr}\left(\pi^{*} \pi\right)$ corresponds to the size of $k S U(n)$ YM instantons in the first hyperplane. Now consider $k S U(n)$ instantons in the first hyperplane. We regard the second diagonal elements of $\left(z_{1}, w_{1}\right)$ as the positions

9 If $\alpha=\beta$, we have

$$
\left[t_{1}, t_{2}\right]=0, \quad\left[t_{1}, t_{1}^{*}\right]+\left[t_{2}, t_{2}^{*}\right]=0
$$

which is the ADHM description of $N$ point-like instantons on 4-dimensional hyperplane defined by $t_{3}=t_{4}=0, t_{5}=\alpha$. 
of $k$ D-instantons in the first hyperplane (the half of the trace is the center mass). The off-diagonal elements of $\left(z_{1}, w_{1}\right)$ can be interpreted as string joining the $k$ D-instantons confined in the first hyperplane. 10

Now we consider the degenerate instantons in the first hyperplane. For example, we consider the case that $\ell=2$. Then the matrices $z_{1}$ and $w_{1}$ become

$$
\begin{array}{rlr}
z_{1}=\left(\begin{array}{cc}
z_{1}^{\prime} & 0 \\
0 & z_{1}^{\prime \prime}
\end{array}\right), & z_{1}^{\prime \prime}=\left(\begin{array}{cc}
a_{1} & 0 \\
0 & a_{2}
\end{array}\right), \\
w_{1}=\left(\begin{array}{cc}
w_{1}^{\prime} & 0 \\
0 & w_{1}^{\prime \prime}
\end{array}\right), & w_{1}^{\prime \prime}=\left(\begin{array}{cc}
b_{1} & 0 \\
0 & b_{2}
\end{array}\right),
\end{array}
$$

and, from (5.4), we have

$$
\sigma=\left(\begin{array}{c}
\sigma^{\prime} \\
0 \\
0
\end{array}\right), \quad \pi=\left(\begin{array}{lll}
\pi^{\prime} & 0 & 0
\end{array}\right)
$$

where $z_{1}^{\prime}, w_{1}^{\prime}$ are $(k-2) \times(k-2)$ matrices, $\sigma^{\prime}$ and $\pi^{\prime}$ are $(k-2) \times n$ and $n \times(k-2)$ matrices, respectively. From (5.4), we have

$$
\begin{aligned}
& {\left[z_{1}^{\prime}, w_{1}^{\prime}\right]+\sigma^{\prime} \pi^{\prime}=0,} \\
& {\left[z_{1}^{\prime}, z_{1}^{\prime *}\right]+\left[w_{1}^{\prime}, w_{1}^{\prime *}\right]+\sigma^{\prime} \sigma^{\prime *}-\pi^{\prime *} \pi^{\prime}=0 .}
\end{aligned}
$$

Now we have two point-like instantons whose positions are given by the eigenvalues $\left(a_{1}, b_{1}\right)$ and $\left(a_{2}, b_{2}\right)$ together with $k-2 S U(n)$ non-degenerated instantons. The form of the matrices above clearly show there are no-strings joining the genuine point-like instantons with the $S U(n) k-2$ instantons. If we look at the second column of the equation (5.4) describing the instantons on the second hyperplane, we find by examining $\pi$ that they describe $S U(k-2), n$ instantons.

A similar analysis can be repeated for more general solutions. For a given partition $N=\sum k_{i}$ with $i=1, \ldots, n_{p}$ we have $k_{i}$ D-instantons confined in $i$-th 4 -dimensional hyperplane defined by $t_{3}=t_{4}=0, t_{5}=\alpha_{i}$.

Up to now we have considered the supersymmetric ground states after breaking half of the supersymmetry. Now we consider the perturbation breaking one quater of the supersymmetry (Sect. 4.1.1). This leads to resolution of singularities. The singularities occur in the moduli space because we allow the instantons degenerate. For example the

10 Of course, the above description is well defined modulo $U(k)$ symmetry. Strictly speaking, the positions of D-instantons (equivalently point-like instantons) in the first hyperplane is not well-defined. Only the center of mass given by $\left(\frac{1}{k} \operatorname{tr} z_{1}, \frac{1}{k} \operatorname{tr} w_{1}\right)$ is well-defined. 
system described by (5.6) and (5.7) corresponds to the 2 nd lower strata $\mathcal{M}_{n, k-2} \times S^{2}\left(\mathbb{C}^{2}\right)$ of the completed moduli space $\overline{\mathcal{M}}_{n, k}$. The 2 nd lower strata $\mathcal{M}_{n, k-2} \times S^{2}\left(\mathbb{C}^{2}\right)$ has singularities when positions of two points coincides. One the hand, the perturbed system does not allow solutions like (5.6) with $a_{1}=a_{2}$ and $b_{1}=b_{2}$. Here it is more convenient to consider the real perturbation (4.20). Then we discover off-diagonal components like

$$
z_{1}^{\prime \prime}=\left(\begin{array}{cc}
a_{1} & \alpha \\
0 & a_{1}
\end{array}\right), \quad w_{1}^{\prime \prime}=\left(\begin{array}{cc}
b_{1} & \beta \\
0 & b_{1}
\end{array}\right)
$$

with the case $(\alpha, \beta)=(0,0)$ excluded [27]. Mathematically such a perturbation resolves the singularities in the completed moduli space of instantons. Physically, we get a new massless degree of freedom due to $(\alpha, \beta)$.

So far we have demonstrated that our extended monads interpolate between Dinstantons and the Yang-Mills instantons in very interesting way. We may identify the extended monads with D-instantons and Yang-Mills instantons as its special sub-sector. Note that those complex 2-dimensional hyperplanes, where the Yang-Mills instantons are living, are derived notions.

5.2. Monads, Space-time, Reciprocity, D-branes and Universal Instantons; Some conjectural discussions

We saw that certain special configurations of D-instantons can be identified with YangMills instantons. An important point is that the identification has been made via the ADHM description, which is the Fourier-Nahm transformation of instantons on $\mathbb{R}^{4} / H$ with $H=\{p t\}$. Some properties, the reciprocal relation and the isometry, of the ADHM description of Yang-Mills instantons has an almost perfect analogy with the Fourier transformation. Abusing terminology, we will refer those properties collectively as weak reciprocity. It is know that weak reciprocity holds for Yang-Mills instantons with an arbitrary $H$. We may use the term strong reciprocity if the dual connections defined by the ADHMN data in the dual (momentum) space are also instantons. For general $H$ strong reciprocity does not hold. A notable exception is the case of a flat torus where the Nahm transformation leads to instantons on the dual torus with the rank of gauge group and the instanton number exchanged [51] [7].

On the other hand, strong reciprocity does not hold for instantons on $\mathbb{R}^{4}$. This is essentially due to the non-compactness of $\mathbb{R}^{4}$, which requires special asymptotic condition (framing) at infinity for the existence of solutions. If we remove such framing, strong reciprocity holds. Consider $k$ number of point-like instantons (completely degenerated 
Yang-Mills instantons with any gauge group) in $\mathbb{C}^{2}$. The monad (ADHM) description of such instantons is

$$
\begin{aligned}
{\left[\tau_{1}, \tau_{2}\right] } & =0, \\
{\left[\tau_{1}, \tau_{1}^{*}\right]+\left[\tau_{2}, \tau_{2}^{*}\right] } & =0,
\end{aligned}
$$

where $\tau_{1}$ and $\tau_{2}$ are $U(k)$-valued matrix. Let $g \in U(k)$, we have the adjoint $U(k)$ symmetry $\left(\tau_{1}, \tau_{2}\right) \rightarrow\left(g \tau_{1} g^{-1}, g \tau_{2} g^{-1}\right)$. One can form the dual connection $\tilde{A}$ with complex covariant derivatives $\tilde{D}_{1}$ and $\tilde{D}_{2}$ in the dual space $\left(\mathbb{C}^{2}\right)^{*}$. From $(5.10)$, we have

$$
\begin{aligned}
F_{\tilde{A}}^{0,2} & =\left[\tilde{D}_{1}, \tilde{D}_{2}\right]=0, \\
\Lambda F_{\tilde{A}}^{1,1} & =\left[\tilde{D}_{1}, \tilde{D}_{1}^{*}\right]+\left[\tilde{D}_{2}, \tilde{D}_{2}^{*}\right]=0,
\end{aligned}
$$

which shows that the $U(k)$ connection $\tilde{A}$ is self-dual in $\left(\mathbb{C}^{2}\right)^{*}$. Now we can view the ADHM description of the $k$ point-like instantons as the dimensional reduction of the above equation for $U(k)$ instantons on the momentum space down to zero-dimension.

We recall that the ADHM matrices $T_{i}$ are related with the coordinates $x_{i}$ of $\mathbb{R}^{4}$ by [5] [10]

$$
T_{i}=-\int x_{i} \psi^{\dagger} \psi d^{4} x
$$

where $\psi$ denotes normalizable cokernel of Dirac operator in the instanton background. For example [26], to any solution of the ADHM equations for $k$ instantons we can associate centre of mass in $\mathbb{R}^{4}$ with coordinates $\left\{x_{i}=\frac{1}{k} \operatorname{tr} T_{i}\right\}$. When the matrices $T_{i}$ commute (for point-like instantons), one can interpret them as space-time coordinates (positions of pointlike instantons). It is clear that the ADHM description of points (point-like instantons) is highly redundant. The off-diagonal elements of $T_{i}$ start to play an important role when we want to resolve the singularities in $S^{k}\left(\mathbb{R}^{4}\right)$. Mathematically, one interprets the moduli space of rank 1 torsion-free sheaves 11 on $\mathbb{R}^{4}$ as natural resolution of $S^{k}\left(\mathbb{R}^{4}\right)$ [27]. In the standard physical interpretation, a singularity is just a sign of new new massless degree of freedom which can be resolved by an extension, for example, as described by (5.9). The most natural explanation of such a degree of freedom can be found by replacing the point-like instantons with D-instantons. A D-instanton has $U(1)$ degree of freedom due to open string which becomes massless when two D-instantons coincide. Thus, the ADHM description is naturally directing us to find a new geometry relevant to D-branes.

In the previous section, the appearance of the genuine ADHM description of YangMills instantons as a special sector of D-instantons has motivated us to interpret the

11 The original moduli space of $k U(1)$ instanton moduli space is empty. However, the completed moduli space after resolution of singularities is non-empty and identical to length $k$ Hilbert scheme of points on $\mathbb{R}^{4}$. 
off-diagonal components of $T_{i}$, in its generalized form to 10-matrices, as representing open strings. As a summary, one may interpret the Nahm transformation (5.19) as a map which relates a gauge field theoretic description in the usual geometry to a stringy one in a non-commutative geometry. These considerations naturally lead us to conjecture of the existence of certain universal instantons, which may be responsible for the D-brane physics.

As we mentioned in the introduction, the Fourier-Nahm transformation can be generalized to Yang-Mills instantons on $\mathbb{R}^{4} / H$ with any subgroup of isometries on $\mathbb{R}^{4}[5]$. Note that the effective theory of $N$ D-instantons is $\mathcal{N}=1$ super-Yang-Mills theory in $d=10$ with $U(N)$ gauge group translation invariant in all directions. In other words, it is the super-Yang-Mills theory on $\mathbb{R}^{* 1,9}$ with isometry group $\mathbb{R}^{* 1,9}$. Now we want to identify the isometry group $\mathbb{R}^{* 1,9}$ with the dual $H^{*}$ of the isometry group $H=\{p t\}$ of the dual space $\mathbb{R}^{1,9}$. In other words, we identify the $U(N)$ connections of $D=10 \mathrm{SYM}$ theory as the 10 matrix functions of $\mathbb{R}^{* 1,9} / H^{*}$ obtained by a Fourier-Nahm transformation of $N$ number of certain instantons in $\mathbb{R}^{1,9}$ with isometry group $H=\{p t\}$. Note that the SYM theory is embedded in the space of ADHM data. Note also that the number of D-instantons is exchanged with the rank of the gauge group. This naturally suggests that the usual description of D-instantons via Witten's non-commutative geometry can be viewed as a Fourier-Nahm transformation of $N$ point-like instantons in $\mathbb{R}^{1,9}$ with isometry group $H=\{p t\}$. The effective theory of $N \mathrm{D} p$-branes, $p>-1$ is $\mathcal{N}=1$ SYM theory in $\mathbb{R}^{* 1,9}$ with isometry group $H^{*}=\mathbb{R}^{0,9-p}$. One may interpret this as a Fourier-Nahm transformation of $N$ instantons in $\mathbb{R}^{1,9}$ with isometry group $H=\mathbb{R}^{p+1}$. That is, the $N$ $p$-branes are described by 10 number of $U(N)$-matrix functions of $\mathbb{R}^{* 1,9} / H^{*}$.

The above suggestion leads us to some general questions;

(1) What is the real space-time? One may regard the usual ten-dimensional space-time $R^{1,9}$ as an auxiliary space. According to this view-point, the arena of the string theory is the space of ADHMN-like data (monads) which encodes information of various D-branes and their excitations (strings) as well as the interactions. The other possible viewpoint is to regard the usual space-time more fundamental. On that spacetime, there may exist universal instantons and string theory can be understood as a way of solving problems by after Fourier-Nahm-like transformation. We may regard the conjectural existence of the universal instantons and reciprocity as a first principle. This seems to suggest that it may be fruitable to regard the both spaces as one object.

(2) Note that (5.10) is a special limit of (2.6), where the reciprocity becomes obscure due to the framing (the source term) at the infinity [10] [5]. In the ADHM construction, the source term encodes the data of the gauge group. This suggests an interesting possibility. The hypothetical instanton reciprocal to the D-instanton may be the completely degenerate 
(or the zero-size) limit of certain universal instantons. In that case, we can expect a source term (or framing at the infinity in 10-dimensions) in the RHS of (5.1). Our discussions in the previous sections suggest that the most natural origin of such a source term is the extra dimensions. This is an exciting possibility related with the $F$-theory of Vafa [52]. If this conjecture turns out to be valid, we may expect to have a natural explantion of the additional 2-dimensions which is responsible to the $S L(2, \mathbb{Z})$ duality of type IIB strings.

(3) The analogy with the Nahm transformation of instantons on the flat 4-torus suggests that reciprocity may be identified with $T$-duality. Related suggestions have already been made in refs. [21]24]. In this paper we concentrated on D-branes in flat space-time. Recently, Taylor studied D-branes on a torus [53]. It will be interesting to generalize our case to curved space.

(4) Recently, it has been suggested [40] that $M$-theory [41] may be realized as the $N \rightarrow \infty$ limit of $p=0$ D-branes [54, related tp 11-dimensional supermembrane [55]. The obvious counterpart for $F$-theory side is the $N \rightarrow \infty$ limit of D-instantons. According to reciprocity, the matrix model $\mathrm{M}$-theory may be regarded as a special case with isometry group $H=\mathbb{R}$.

Finally, we would like to point out some issues concering the model we considered in the paper. We used the set of equations (4.1) (4.3), which dimensional reduction of the Vafa-Witten equations (4.6), as a model for D-instantons. The same set of equations can be obtained by reducing a self-duality equation in Calabi-Yau 4-folds (or $\mathbb{R}^{8}$ ) introduced by Donaldson and Thomas [56]:

$$
F_{+}^{0,2}=0, \quad F \cdot \omega=0
$$

where $\omega$ is the Kähler form. Here, $F_{+}^{0,2}$ denote self-dual part of $(0,2)$-component of curvature with respect to the norm

$$
\left(\alpha^{0,2}, \alpha^{0,2}\right)=\left|\alpha^{0,2}\right|^{2} \wedge \overline{\omega^{0,4}}
$$

where $\omega^{0,4}$ denotes the non-degenerated holomorphic 4-form. The natural origin of the Vafa-Witten type equations in 8-dimensional manifolds has been pointed out in [57]. It will be very interesting to study Fourier-Nahm transformations of (5.13). This may be a good place to test the conjecture in (2) above. The set of equations (4.1) (4.3) may be appear as a completely degenerate limit of ADHM like construction in $\mathbb{R}^{8}$. It seems to be reasonable to expect that Fourier-Nahm transformation of (5.13) has reciprocity relating mirror Calabi-Yau 4-folds. This conjecture is motivated by the quantum mirror symmetry as $T$-duality of $T^{4}$ fibration [58].

Clearly, there remain many questions and unsolved problems requiring further study. 


\section{Acknowledgments}

I am very grateful to R. Dijkgraaf and B. J. Schroers for collaboration on a related subject and for useful discussions, and to B. J. Schroers for introducing me the ADHMN construction and reading this manuscript. I would like to thank F. A. Bais, F. Hacquebord and Q-Han Park for useful discussions. I am grateful to H. Nakajima for sending me his papers and the preliminary version of lecture notes, for useful communications and pointing out an error. Special thanks to H. Verlinde for many useful discussions, suggestions and encouragement throughout this project. This work is supported by a pioneer fund of NWO. 


\section{References}

[1] A. Belavin, A. Polyakov, S. Schwartz and Y. Tyupkin, Psseudoparticle solutions of the Yang-Mills equations Phys. Lett. B59 (1975) 85

[2] M. Atiyah, V. Drinfeld, N. Hitchin and Yu. Manin, Construction of instantons, Phys. Lett. 65A (1978) 185

[3] R. S. Ward, Phys. Lett. 61A (1977) 81 ;

M. F. Atiyah and R.S. Ward, Instantons and algebraic geometry Comm. Math. Phys. 55 (1977) 117

[4] S. K. Donaldson, Instantons and geometrical invariant theory, Comm. Math. Phys. 93 (453) 1984

[5] W. Nahm, Multimonopoles $i$ the ADHM construction, in "Proc. Symp. on particle physics", eds. Z. Horvath et al., (Vsiegrad, 1981); Construction of all self-dual monopoles by the ADHM method, in "Monopoles in quantum field theory". eds. N. Craigie et al., (World Scientific, Singapore, 1982); Self-dual monopoles and calorons, in Lecture Notes in Physics, vol. 201, eds. G. Denardo et al., (Springer 1984)

[6] S. Mukai, Dulaity between $D(X)$ and $D(\hat{X})$, with application to Picard sheaves, Nagoya Math. J. 81 (1981) 153.

[7] P. J. Braam and P. van Baal, Nahm's transformation for instantons, Comm. Math. Phys. 122 (1989) 267

[8] A. Maciocia, Metrics on the moduli spaces of instantons over Euclidean 4-space, Comm. Math. Phys. 135 (1991) 467

[9] H. Nakajima, Monopoles and Nahm's equations, in Einstein metric and Yang-Mills connections, eds. T. Mabuchi and S. Mukai, (Marcel Dekker, Inc. 1993)

[10] E. Corrigan and P. Goddard, Construction of instanton and monopoles solutions and reciprocity, Ann. Phys. 154 (1984) 253

[11] J. Polchinski, Dirichlet-Branes and Ramond-Ramond Charges, hep-th/9510017;

J. Polchinski, S. Chaudhuri, C.V. Johnson, Notes on D-Branes hep-th/9602052

[12] M.R. Douglas, Superstring dualities, Dirichlet branes and the samll scale structure of space, hep-th/9610041.

[13] J. Dai, R. G. Leigh, J. Polchinski, New connections between string theories, Mod. Phys. Lett. A4 (1989) 2073

[14] E. Witten, Bound states of strings and p-branes, hep-th/9510135

[15] C. Vafa and E. Witten, A strong coupling test of S-duality, Nucl. Phys. B 431 (1994) 3 . 
[16] R. Dijkgraaf, J.-S. Park and B. J. Schroers, to appear.

[17] E. Witten Small instantons in string theory, hep-th/9511030

[18] E. Witten, Sigma models and the ADHM construction of instantons, hep-th/9410052

[19] M. R. Douglas Branes within Branes, hep-th/9512077. ; Gauge Fields and D-branes, hep-th/9604198.

[20] P. B. Kronheimer and H. Nakajima, Yang-Mills instantons on ALE gravitational instantons, Math. Ann. 288 (1990) 263

[21] M. R. Douglas, G. Moore D-branes, Quivers, and ALE Instantons, hep-th/9603167

[22] C. Johnson and R. Myers, Aspects of type IIB theory on ALE spaces, hep-th/9610140.

[23] K. Furuuchi, H. Kunitomo and T. Nakatsu, Topological field theory and secondquantized five-branes, hep-th/9610016.

[24] D.-E. Diaconescu, D-branes, Monopoles and Nahm Equations, hep-th/9608163

[25] M. F. Atiyah, Geometry of Yang-Mills fields, Scuola Normale Superiore, Pisa, 1979.

[26] S. K. Donaldson and P.B. Kronheimer, The geometry of four-manifolds, Oxford Mathematical Monographs, Clarendon Press, Oxford, 1990

[27] H. Nakajima, Lectures on Hilbert schemes of points on surfaces, Lecture Notes given at Dep. of Math. Sci., Univ. of Tokyo, to appear.

[28] G. Horrocks, Vector bundles on the punctured spectrum of a local ring, Proc. London Math. Soc. 14 (1964) 684

[29] W. Barth, Some properties of stable rank-2 vector bundles on $P_{n}$, Invent. Math. 42 (1977) 63;

W. Barth and K. Hulek, Monads and moduli of vector bundles, Manuscripta Math. 25 (1978) 125.

[30] S. K. Donaldson, Anti-self-dual Yang-Mills connections on complex algebraic surfaces and stable vector bundles, Proc. London Math. Soc. 3 (1985) 1;

Infinite determinants, stable bundles and curvature, Duke Math. J. 54 (1987) 231

[31] S. Hyun and J.-S. Park, N=2 topological Yang-Mills theories and Donaldson's polynomials, J. Geom. Phys. 20 (1996) 31, hep-th/9404009

[32] H. Nakajima, Instantons on ALE spaces, quiver varieties, and Kac-Moody algebra, Duke Math. J. 76 (1994) 365; Homology of moduli spaces of instantons on ALE spaces. I, J. Differ. Geom. 40 (1994) 105; Instantons and affine Lie algebras, alg-geom/9510003

[33] R. Dijkgraaf, G. Moore, Balanced Topological Field Theories, hep-th/9608169

[34] E. Witten, Monopoles and four-manifolds, Math. Research Lett. 1 (1994) 769. 
[35] F. Kirwan, Cohomology of quotients in symplectic and algebraic geometry (Princeton Univ. Press, 1987).

[36] J. J. Duistermaat and G. J. Heckman, On the variation in the cohomology of the symplectic form of the reduced phase space, Invent. Math. 69 (1982) 259; Addendum, Invent. Math. 72 (1983) 153.

[37] V. Guillemin and S. Sternberg, Birational equivalence in symplectic category, Invent. Math. 97 (1989) 485.

[38] E. Witten Two dimensional gauge theories revisited, J. Geom. Phys. 9 (1992) 303 hep-th/9204083

[39] L.C. Jeffrey and F.C. Kirwan, Localization for non-abelian group actions, preprint alg-geom/9307005.

[40] P. Townsend, D-branes from M-branes, Phys. Lett. B373 (1996) 68;

T. Banks, W. Fischler, S. H. Shenker and L. Susskind, M theory as a matrix model: a conjecture, hep-th/9610043;

M. Berkooz and M. Douglas, hep-th/9610236;

V. Periwal, Matrics on a point as the theory of everything, hep-th/9611103

[41] M. J. Duff, P. Howe, T. Inami, K.S. Stelle, Superstrings in D=10 from supermembranes in $D=11$, Phys. Lett. B191 (1987) 70;

M. J. Duff, J. X. Lu, Duality rotations in membrane theory, Nucl. Phys. B347 (1990) 394 ;

M. J. Duff, R. Minasian, James T. Liu, leven-dimens ional origin of string/string duality: a one-loop test, Nucl. Phys. B452 (1995) 261 ;

C. Hull and P. K. Townsend, Unity of superstring dualities, Nucl. Phys. B438 (1995) 109, ;

P. K. Townsend, The Eleven-Dimensional Supermembrane Revisited, Phys. Lett. B350 (1995) 184; String-membrane duality in seven dimensions, Phys. Lett. 354B (1995) 247, ;

C. Hull and P. K. Townsend, Enhanced gauge symmetries in superstring theories, Nucl. Phys. B451 (1995) 525, ;

E. Witten, String Theory Dynamics in Various Dimensions, Nucl. Phys. B443 (1995) 85

[42] J.-S. Park, Monadic Yang-Mills theory, in preparation.

[43] S. K. Donaldson, Polynomial invariants for smooth 4-manifolds, Topology 29 (1990) 257.

[44] E. Witten, Topological quantum field theory, Comm. Math. Phys. 117 (1988) 353; Introduction to cohomological field theories, Int. J. Mod. Phys. A6 (1991) 2273 
[45] G. 't Hooft, A planar diagram theory for strong interactions, Nucl. Phys. B72 (1974) 461

[46] H. Nakajima, Gauge theory on resolutions of simple singularities and simple Lie algebras, Int. Math. Res. Not. 2 (1994) 61; Gauge theory on resolutions of simple singularities and affine Lie algebras; Resolutions of moduli spaces of ideal instantons on $\mathbb{R}^{4}$, in "Topology, geometry and field theory", World Scientific, (Singapore, 1994).

[47] E. Brézin and S. R. Wadia, eds., The large $N$ expansion in quantum field theory and statistical physics; from spin systems to 2-dimensional gravity, World Scientific, (Singapore, 1993).

[48] J.-S. Park, N=2 topological Yang-Mills theory on compact Kähler surfaces, Comm. Math. Phys. 163 (1994) 113; Holomorphic Yang-Mills theory on compact Kähler manifolds; Nucl. Phys. B423 (1994) 559

[49] H. Nakajima, private communication.

[50] H. Nakajima, Heisenberg algebra and Hilbert schemes of points on projective surfaces, alg-geom/9507012

[51] H. Schenk, On a generalized Fourier transformation of instantons over flat tori, Comm. Math. Phys. 116 (1988) 183

[52] C. Vafa, Evidence for F-theory, hep-th/9602022.

[53] W. Taylor IV, D-brane field theory on compact spaces, hep-th/9611042

[54] U. H. Danielsson, G. Ferretti and B. Sundborg, D-particle Dynamics and Bound States, hep-th/9603081;

D. Kabat and P. Pouliot, A Comment on Zero-Brane Quantum Mechanics, hepth/9603127;

M. R. Douglas, D. Kabat, P. Pouliot and S. Shenker, D-Branes and Short Distance in String Theory, hep-th/9608024.

[55] B. de Wit, J. Hoppe and H. Nicolai, Nucl. Phys. B305 (1988) 545; Nucl. Phys. B320 (1989) 135.

[56] S. Donaldson, Lectures given at I. Newton Inst., Nov. 1996.

[57] M. Bershadsky, V. Sadov and C. Vafa, D-branes and topological field theories, Nucl. Phys. B463 (1996) 420.

[58] A. Strominger, S.-T. Yau and E. Zaslow, Mirror symmetry is T-duality, hepth/9606040;

D. R. Morrison, The geometry underlying mirror symmetry, alg-geom/9608006;

K. Becker, M. Becker, D.R. Morrison, H. Ooguri, Y. Oz and Z. Yin, Supersymmetric cycles in exceptional holonomy manifolds, hep-th/9608116. 